\title{
The place of Metropolitan France in the European genomic landscape
}

Simone Andrea Biagini ${ }^{1}$, Eva Ramos-Luis ${ }^{2,3}$, David Comas ${ }^{1}$, Francesc Calafell ${ }^{1 *}$

1. Departament de Ciències Experimentals i de la Salut, Institute of Evolutionary Biology (CSIC-UPF), Universitat Pompeu Fabra, Barcelona, Catalonia, Spain

2. Xenética Cardiovascular, Instituto de Investigación Sanitaria de Santiago de Compostela, Complexo Hospitalario Universitario de Santiago de Compostela, Santiago de Compostela, A Coruña, Spain.

3. Grupo de Medicina Xenómica, Universidade de Santiago de Compostela- Fundación Pública Galega de Medicina Xenómica, Santiago de Compostela, A Coruña, Spain.

* Address correspondence to Francesc Calafell, francesc.calafell@upf.edu, Departament de Ciències Experimentals i de la Salut, Institute of Evolutionary Biology (CSIC-UPF), Universitat Pompeu Fabra, Carrer Doctor Aiguader 88, 08005 Barcelona, Catalonia, Spain. 


\section{Abstract}

Unlike other European countries, the human population genetics and demographic history of Metropolitan France is surprisingly understudied. In this work, we combined newly genotyped samples from various zones in France with publicly available data and applied both allele frequency and haplotype-based methods in order to describe the internal structure of this country, by using genome-wide single nucleotide polymorphism (SNP) array genotypes. We found out that French Basques, already known for their linguistic uniqueness, are genetically distinct from all other groups and that the populations from southwest France (namely the Gascony region) share a large proportion of their ancestry with Basques. Otherwise, the genetic makeup of the French population is relatively homogeneous and mostly related to Southern and Central European groups. However, a fine-grained, haplotype-based analysis revealed that Bretons slightly separated from the rest of the groups, due mostly to gene flow from the British Isles in a time frame that coincides both historically attested Celtic population movements to this area between the 3th and the 9th centuries CE, but also with a more ancient genetic continuity between Brittany and the British Isles related to the shared drift with hunter-gatherer populations. Haplotype-based methods also unveiled subtle internal structures and connections with the surrounding modern populations, particularly in the periphery of the country.

Keywords: France, population history, gene flow, haplotype-based methods 


\section{Introduction}

Located in the center of Western Europe, Metropolitan France has historically acted as a bridge connecting Northern Europe to the Mediterranean and the Iberian spaces. So far, similar studies have produced valuable information about the history of other European countries (Leslie et al. 2015; Bycroft et al. 2019; Raveane et al. 2019), while France still remains poorly studied. The geographical position of France strongly affected the history of the settlement of the different parts of the territory, whose continuous fragmentation through time is attested by the large number of populations and cultures that settled this area. Greeks, Romans and Celtic tribes from central Europe shaped a first internal structure between the 6th and the 1st centuries BCE, while waves of barbarian invasions (Alamanni, Burgundians, Visigoths, Franks, and Celts) strongly impacted the population landscape of France during the 5th century CE (Haine 2000). During the 9th and 10th centuries $\mathrm{CE}$, foreign invasions from all sides also influenced the territory: Muslims and Saracens from North Africa coming through Iberia, Hungarian Magyar from the east, and Vikings (Northmen) from the north (Haine 2000). Nowadays, France is a cosmopolitan country whose society is shaped by a plurality of lifestyles and ethno-cultural diversity. Without any doubt, the modern genetic landscape of the French territory has been enriched by recent events. Among them, the impact of political refugees throughout the 20th century, or of the immigration from colonized countries to mainland France, such as the migration of Arabs and Berbers from Algeria which was the most extensive of all colonial migrations to Western Europe before the 1960s (MacMaster 1997). However, it is beyond our intention to explore this plethora of recent genetic contributions here, which can be quantified much more precisely with demographic analyses. Instead, we can apply genomic tools to uncover a more ancient genetic background. At the light of this complex past, the genetic landscape of France has been poorly analyzed, especially in recent times. Previous works agreed on a general homogeneity for the genetic landscape of continental France. Results were similar when considering autosomal variants (Kherumian et al. 1967; Cambon-Thomsen and Ohayon 1988; Karakachoff et al. 2015), mitochondrial DNA (Dubut et al. 2004; Richard et al. 2007), and Y chromosome (Ramos-Luis et al. 2009). Furthermore, Brittany was generally identified as an outlier in some of these studies (Dubut et al. 2004; Richard et al. 2007; Ramos-Luis et al. 2009; Karakachoff et al. 2015) In this work, we present a comprehensive genome-wide study on France, using both allele frequency and haplotype-based methods. 
Our first goal is to determine the best geographical level of genetic stratification within France and arrange our samples according to this finding. After the French Revolution in 1790, in order to weaken the old loyalties, the ancient provinces of France were subdivided into departments, whose overall configuration has been mostly conserved so far (Forstenzer 2016). Furthermore, in 1982, a system of 22 regions was established by grouping different departments into wider areas (Sowerwine 2009). However, in 2016, the number of the regions was reduced to 13 , with the consequent rearrangement of the departments (OECD 2017). Secondly, we aim to describe the geogenetical landscape patterns within France, searching for internal levels of stratification. Lastly, we aim to trace the historic and ancient sources of gene flow into mainland France, taking into account both modern and ancient contributions from external sources.

\section{Results}

In agreement with the type of analyses we carried out, the results we present in this section refer to different sample compositions and number of variants used. Supplementary table 1 provides a complete description for the set-up of the analyses we made, together with a full description in the materials and methods section. For clarity, each of the following paragraphs will also start with a statement on the number of samples and variants used, unless the same dataset from the previous paragraph is still being analysed.

\section{Internal genetic structure in France}

This analysis involved 395 samples and 142,803 variants (Dataset A). In order to define the best geographical partitioning of genetic differentiation, a hierarchical analysis of molecular variance (AMOVA) was performed with areas or regions as major grouping factors. We determined first the proportion of genetic variation partitioned among geographic areas, among departments within geographic areas, and within departments. We next tested the proportion of genetic variation partitioned among regions (considering the 13 regions established in 2016), among departments within regions, and within departments. A further AMOVA was performed testing only the proportion of genetic variation partitioned among and within departments. As shown in Table 1, in all cases the 
main contribution to the genetic variance was found at the lowest hierarchical level (variation within departments), while differences among regions resulted in a negative value that could be interpreted as zero, meaning absence of any structure at this level. Conversely, differences among areas displayed positive, although not statistically significant, values, supporting the role of areas as more reliable grouping factors of genetic variations when considering wider sample distributions. Finally, the results for the variation between departments, show small, albeit statistically significant values in all the AMOVA analyses; this finding might point to the fact that this level of stratification might be considered as a better representation for the minimal unit of genetic differentiation. Based on these results, samples were distributed on the map according to the departmental locations (Supplementary Figures 1-4 and Supplementary Table 1) and all the subsequent analyses considered this grouping factor, although, given their known cultural and genetic identity, we retained Basque-speakers as a separate group in the Pyrénéés-Atlantiques department. The extended data table provides a complete information about sample size distribution per department, regions, and areas.

Using departments as minimal unit of genetic differentiation, a first Principal Component Analysis (PCA) showed two distinct groups separated along the first PC (Figure 1A): the Basque samples on the right part of the plot, against most of the rest of the samples on the left one, within which a structure cannot be defined. These two major groups are connected by a "bridge" of samples represented by non-Basque-speaking individuals from the Gascony region in the southwestern corner of France. When we averaged the eigenvalues for the first two PCs and represented the same PCA, together with standard deviation (SD) values for each group, no evident pattern could still be discerned beyond the separation of Basques and Gascons (Supplementary Figure 5A). When we removed both Basque and Gascon samples from the analysis (Figure 1B), the resulting PCA showed some internal pattern of differentiation, more clearly defined by the average PCA (Supplementary Figure 5B), in which samples from the departments belonging to the northwestern region of Brittany seem to form a cluster on the left part of the plot.

\section{Patterns of gene flow within France}

To highlight patterns of internal differentiation we used the EEMS analysis, a method for visualizing genetic diversity patterns and explicitly model the spatial structure in the data. 
We noticed that a higher effective migration was locally found in northern, northeastern and northwestern France among departments belonging to the same geographical areas, while a major barrier was discovered along the western side of France. We observed that the resulting effective migration surface mirrors the outcomes of genetic differentiation detected by the $F_{S T}$ analyses (Figure 2). Indeed, in the genetic variation computed with the $F_{S T}$ analysis, a general homogeneous pattern was found, with fine scale values of differentiation between some departments. In general, among Europeans, low average levels of genetic differentiation are recorded, with values increasing with geographic distances (Novembre et al. 2008). Normally, $F_{S T}$ values tend to be smaller between population with origins in neighboring regions $\left(F_{S T}=0.001\right)$, while higher values are recorded for more distant groups $\left(F_{S T}=0.020\right)$ (Tian et al. 2009). In our analysis, the southwestern samples (Basques and Gascons) showed the highest values of differentiation with the northwestern departments reaching scores between 0.008 and 0.009 for the Basque-speaking samples, and between 0.004 and 0.006 for the non-Basquespeaking ones (Supplementary Figure 6A, left), followed by lower values of differentiation with the northern and northeastern departments. Without the southwestern samples, the main differentiation was recorded between the northwestern departments and the southeastern corner of the country, with a highest value of differentiation around 0.002 between the southeastern department of Bouches-du-Rhône (BdR) and the northwestern Breton department of Côtes-d'Armor (CdA) (Supplementary Figure 6B, left). Lower levels of differentiation were locally found among the departments in the northwest, and among those in the north together with the northeastern ones. A Multidimensional Scaling analysis (MDS) based on the $F_{S T}$ matrices clearly showed how the southwestern samples separate from the rest of the groups (Supplementary Figure 6A, right), and how the Breton departments do the same once the Gascon and Basque samples are removed (Supplementary Figure 6B, right). A Mantel test of isolation by distance (IBD) between the $F_{S T}$ values and the geographical distances showed a positive and statistically supported correlation $\left(\mathrm{R}^{2}=0.332, \mathrm{P}=0.001\right)$ (Supplementary Figure 7A), moving to even more positive values when the southwestern samples were removed $\left(\mathrm{R}^{2}=0.432, \mathrm{P}=0.001\right)$ (Supplementary Figure 7B).

\section{Haplotype sharing patterns within France}


This analysis involved 395 samples and 343,884 variants (Dataset A). Using haplotypebased methods, we looked for patterns of haplotype sharing, illustrating relations between departments. In this first step, cutting the fineSTRUCTURE tree at the very base, allowed us to describe a fine scale haplotype sharing distribution on a departmental scale; the outcome is a picture of the haplotype configuration within France (Figure 3). The resulting map shows finer-grained detail: we can define at least four distinct groups, plus a more widespread component. In the southwestern corner, the Basque samples clearly separate from the Gascon ones. In the northwestern vertex, the Breton departments exhibit their very own haplotypic signature, in agreement with the lower level of differentiation detected with the $F_{S T}$ analysis and the higher internal effective migration rate detected with EEMS. The same was found for the northern and northeastern departments that display a clearly shared haplotypic configuration. The southwestern department of HauteGaronne (HG) and the southeastern one of Bouches-du-Rhône (BdR) present higher frequencies for some local haplotypes that in other departments reached only lower frequencies. Otherwise, a more generally spread French haplotypic background (light orange in Fig. 3) is found on the north-south axis.

\section{Sources of gene flow into France}

This analysis involved 728 samples and 154,889 variants (Dataset B). We added external sources from the surrounding populations (yellow dots in Supplementary Figure 3) to describe allele-based genomic components with ADMIXTURE (Supplementary Figure 8). The neighboring populations were chosen among those European countries historically and geographically closer to France, in contrast with the wider dataset C which included also populations from more distant worldwide regions. The configuration observed in the ADMIXTURE analysis pointed to a general homogeneous picture. The only exception was represented by the samples belonging to the Breton departments whose configuration was more alike to that in the Irish, Scottish, and English groups. Moving through the different $\mathrm{K}$ ancestral components, this behavior clearly characterizes the northwestern departments, separating them from the rest of the French groups since the very first K ancestral components (Supplementary Figure 8). Thus, we formally tested for admixture events using the f3-statistics with the test groups being the different departments, and the external surrounding populations as sources. We only retained the negative $\mathrm{f} 3$ values for those departments represented at least by two individuals. Results 
are shown in Supplementary Table 2 were only significant Z-scores $<-3$ are reported, while results for those departments passing all the requested filters but with higher Zscore values are shown in Supplementary Table 3. Notably, in 9 departments, a combination of sources that was highly significant was Ireland-Southern Italy.

\section{Haplotype sharing patterns with external sources}

This analysis involved 1527 samples and 380,697 variants (Dataset C). Based on the haplotype sharing with external sources it was possible to redefine the French haplotype configuration. After merging the 395 French samples with the 1132 external ones (Dataset C), we first defined the external groups by silencing France when rerunning ChromoPainter and fineSTRUCTURE. The result was represented by 35 different external groups (Supplementary Figure 9a). Secondly, focusing on our target, we redefined the French internal clusters using the 35 external ones as "continents" when running fineSTRUCTURE (Supplementary Figure 9b). The 13 different clusters we found within France were then represented as separate maps (Figure 4); each map in the figure is a heatmap showing the number of samples falling in the different departments. Out of 13 groups, 10 satisfied the conditions of having at least 10 individuals and a major geographical area with a number of subjects corresponding to more than $50 \%$ of the entire cluster. These conditions allowed us to name each cluster based on the fact that a specific area was more represented than others in terms of sample size. The exclusion of three clusters did not impact the analysis, since only $8.35 \%$ of the French samples were then not included as target in the following analyses with GLOBETROTTER. As in the analysis described in the previous paragraph, even in this case France appeared to be organized in few major areas of interest. As shown in Figure 4, the Northwest presented two main groups (B1 and B2), the Southwest divided in Basque (Bas) and Gascon (G1 and G2) groups, the Northern (CN) and Northeastern (NE) areas, the Southeast (SE), and a central/southwestern part of France (CSW1 and SW). These ten main areas represented the targets for the GLOBETROTTER analysis that we used to describe the ancestry profiles, the admixture events, and their dates.

\section{Ancestry profiles and dating admixture events}


The ancestry profiles are a way to describe the genome of each one of the ten French target as a mixture of the genomes from other groups, without inferring any particular admixture event (Hellenthal 2015). With this analysis, each target is described as a composition of different proportions of haplotype sharing with other sources, excluding the contribution of the group that we want to explain (no self-copying allowed). The results from the application of the nnls algorithm are displayed in Figure 4; on both sides of each target the ancestry profiles are represented as doughnut charts (on the left the results from the NM analysis, on the right the ones for the $\mathrm{M}$ one). The different colors represent proportions of haplotype sharing with specific sources (only contributions above $2.5 \%$ are shown). In the $\mathrm{NM}$ analysis, it is possible to appreciate how the haplotype sharing with other French sources (brown color) represents the highest proportion for all the different targets. When masking the French component, more refined patterns of contributions from external sources are detected. With the only exception of the southwestern targets (G1, G2, and Bas), the remaining ones show a higher contribution from north Italy and Great Britain. Apart from this common signal, it is possible to highlight contributions from those neighboring populations that are more geographically close to specific areas within the French territory. The southwestern targets (G1, G2, and Bas) received more from the Spanish side, the northwestern targets (B1 and B2) share more with the external cluster source named Irish_Scottish (with a proportion of $23.91 \%$ and $18.32 \%$ for the B1 and B2 targets respectively), the northeastern target (NE) is more connected to the external cluster source representing central and eastern European countries (receiving $17.64 \%$ from the source we named Central_Eastern_EU), as also from the NorthernEurope cluster source (which contributes $7.35 \%$ and $5.78 \%$ to the NE and $\mathrm{CN}$ targets, respectively). The southeastern target (SE) is mostly connected to the Italian sources and other Mediterranean countries, and the central/southwestern target (CSW1) clearly received more from both Spain and Italy.

As explained in Supplementary note 1, GLOBETROTTER provided evidence of admixture for 8 out of 10 targets, and for 5 of them we could also describe the dates and the sources of admixture as shown in Supplementary Figure 10. For three targets GLOBETROTTER gave one-date as result, while for the remaining two one-datemultiway was detected. In each case, only one date of admixture was detected; for the one-date groups a single admixing couple of sources was described, while two couples 
of sources were presented in the case of one-date-multiway. For a better interpretation of the results, consider the caption from Supplementary Figure 10.

\section{Relations with ancient populations}

This analysis involved 1687 samples and 163,631 variants (Dataset D). We first explored the position of France in the context of other modern populations, and then we focused on the relation with a set of ancient samples from different periods. In Supplementary Figure 11, panel A shows the PCA with the modern samples; France (white circles) is located in a position that mirrors its geographical situation, in between British, Irish, Mediterranean, central and eastern European samples. In panel B, a set of ancient samples was projected into the modern genetic space. In this second PCA, most of the French individuals are close to the Steppe and the Late Neolithic Bronze Age (LNBA) European samples, with some subjects connecting with the Anatolian Neolithic and the Early Neolithic European groups, and few others with the Europe Middle Neolithic and Chalcolithic (Europe_MNChL) samples. Results from the ADMIXTURE analysis are reported for the lowest cross-validation error detected ( $\mathrm{K}=4$ in Figure 5). At this level, four ancestral components are clearly visible: the hunter-gatherer (HG) ancestry (principally represented by the Scandinavian HG, in pink), Neolithic (mostly Anatolian and then European, in green), the Iran Neolithic (black), and Natufian (purple). Again, the proportion of these components in France is intermediate between those in Southern and Central European groups. It is especially the Natufian component that seems to act as a discriminant factor, not only inside France where it is virtually absent with few exceptions on the Mediterranean side, but mostly among the various modern groups. Ancient contacts with Levant populations such as Phoenicians may have contributed this component. Outgroup f3-statistics in the form of f3(Ancient, X; Mbuti) allowed us to quantify for each $\mathrm{X}$ modern group the amount of shared drift with different ancient populations. Figure 6 shows the outcome for these statistics, with a focus on the shared drift with the three main European ancestral components: Western, Eastern, and Scandinavian hunter-gatherers, European Neolithic farmers, and the European Bronze Age steppe component. In most cases, French populations fit the expected pattern of distribution in the wider panorama of the European area. However, the European Neolithic component seems to be higher in the SW of France, while Brittany carries a 
proportion of HG ancestry that is higher than elsewhere in France but closer to the values in the British Isles.

\section{Discussion}

We have used both allele frequency and haplotype-based methods in order to describe the internal structure of pre-20th century Metropolitan France. While the first yielded a more homogeneous landscape, the latter unveiled patterns of local differentiation with some connections with the surrounding European populations. Furthermore, we explored patterns of genetic affinity with ancestral populations, contextualizing France in the wider European panorama. In previous works about France, samples were differently arranged into the geographical space and no consensus had been reached on what subdivision was more appropriate; apart from the peculiar military districts (Kherumian et al. 1967), historical provinces (Cambon-Thomsen and Ohayon 1988; Richard et al. 2007) and old regions (Ramos-Luis et al. 2014) are the most used so far. Thus, our first goal was to search for the best geographical level of genetic stratification before arranging our samples on a map. Given this background, our AMOVA results provide evidence that regions, as a new internal reorganization, are not a suitable model for the genetic compartmentalization and point to the absence of any contribution to the total genetic variation, possibly implying that regions are separating genetically similar departments into different groups. On the other hand, departments, as result of a more conserved internal geographical structure, represent the best minimal unit of genetic stratification.

\section{Dissecting the Hexagone (Metropolitan France)}

Principal component analysis on allele frequencies revealed the expected Basque differentiation, adding Gascons in SW France as a population closely related to them, while the rest of France appeared relatively homogeneous. However, EEMS results pointed to the existence of other barriers to gene flow, particularly between NW France (Brittany) and the rest, while other areas acted as corridors, in central France and along the $\mathrm{N}$ and NE borders (Figure 2). It should not be excluded, though, that unsampled regions caused some possible artifacts (House and Hahn 2018) and that adding samples from missing areas could change the barriers we detected. It was with fineSTRUCTURE that we could define a fine scale internal subdivision of France (Figure 3). A general 
widespread French haplotypic background moving through the north-south axis was detected (light orange color in Figure 3), reaching all but the two Southwestern groups (Basques and Gascons). Particular haplotype sharing patterns could also be observed along the north and northeast of France, in the southeast, and among the northwestern departments.

In order to understand whether these internal patterns of differentiation are due to recent events or whether they reflect a more ancient history, we relied on different analyses. On one hand, we looked at the relation with modern external populations, exploring both allele-frequency (ADMIXTURE and f3-statistics) and haplotype-based methods (using GLOBETROTTER, we described the ancestry profiles for 10 different French targets, defined by the haplotype sharing with external sources, and provided a date of admixture events for 5 of them). On the other hand, we looked for the relations between modern France and ancestral populations from different times.

\section{France, carrefour of Europe}

An ADMIXTURE plot (Supplementary Figure 8), and a PCA with reference populations (Supplementary Figure 11A) place most French populations as similar to their geographic neighbours, namely the British Isles, Central Europe, Spain and Italy, in accordance with the general observation in Europe of geographic distance as the main predictor of genetic distance (Lao et al. 2008; Novembre et al. 2008). This may explain an apparently surprising outcome of our work: 9 out of 22 distinct targets in $\mathrm{f} 3$ statistics (Supplementary Table 2) we tested against different external sources gave significant results with the lowest Z-scores detected for the same couple represented by the South Italian and Irish sources. Z-scores lower than -3 indicate that our test populations are admixed from sources not necessarily identical but related to the sources we used in the analysis (Lazaridis et al. 2016). Interestingly, these results found support in the outcome from the ancestry profiles we carried out with Dataset C. The ancestry profiles described in Figure 4 are informative of differential migratory patterns (Leslie et al. 2015) into each of the ten French genetic targets. Following the previous results from the f3-statistics, in the $\mathrm{M}$ analysis we found that 7 out of the 10 targets we tested were mostly described by high proportions of haplotype sharing with both Italy and the British Isles. Furthermore, the NM analysis highlighted the presence of a very strong shared French component, possibly 
reflecting the result of a higher intermixing between individuals from the different parts of modern France.

An additional dimension to the central genetic position of France in Western Europe is given by the comparison with a time transect of ancient samples. The ADMIXTURE results for dataset D (Figure 5), as well as the projected PCA (Supplementary Figure 11B) place France again as intermediate between Southern and Central Europe. However, this pattern is locally nuanced, as discussed below. Thus, it appears that France has been operating as a crossroads for human migration in Western Europe since, at least, the Early Neolithic.

\section{Basques and Gascons}

These groups clearly differentiated from the rest of France both with allele frequency and with haplotype-based methods. It is interesting to notice that the presence of two distinct groups in the Southwestern region stressed the outcome of the isolation the Basquespeaking group experienced, splitting from their non-Basque-speaking neighbors from the very same department (PA and PAB groups). This finding is in agreement with their recognized distinct cultural entity (Calafell and Bertranpetit 1994) and their genetic outlier position in the European landscape (Rodríguez-Ezpeleta et al. 2010), as also with the lower internal levels of differentiation we detected with the $F_{S T}$ analysis, and the low effective migration rates evidenced by EEMS, resulting in a barrier to migration in the southwestern corner of France.

The ancestry profile for French Basques (Figure 4) reflects an almost exclusive component from Spanish Basques, with some minor contribution from two other source clusters in the Iberian Peninsula. Quite often, Spanish populations are modelled as the result of a Basque background plus external admixture (Bycroft et al. 2019), so it is not surprising that haplotypes found in Basques are also present in Spain. French and Spanish Basques, as well as other populations in NE Iberia, share also an increase in shared drift with Early Neolithic ancient samples (Figure 6D). The Basque singularity has often been explained as due to the persistence of an ancient gene pool, as old as the Late Glacial (Pereira et al. 2005), or as the Pre-Neolithic (Calafell and Bertranpetit 1993), or as the Neolithic (Günther et al. 2015) (as our results seems to suggest), but a recent analysis of 
a large number of ancient Iberian samples (Olalde et al. 2019) points to a more recent divergence, probably in the Iron Age, of the Basque population.

Gascons have been shown to be intermediate between French Basques and other French populations by PCA (Figure 1), and to carry a sizable proportion of Basque ancestry (Figure 4). This could be the result of the postulated contraction of the Basque-speaking lands since the late Antiquity. Place names may indicate that Basque or languages similar to it may have been spoken in Aquitaine (SW France) south of the Garonne river (Zuazo 2010).

\section{The Celtic connection}

As shown by EEMS (Figure 2), a barrier to gene flow delineates the northwestern corner of France, indicating the presence of another distinct group represented by the Breton departments. This group was firstly detected, on a coarser scale, with the removal of the Southwestern samples (Basques and Gascons) from the first PCA, and its outstanding position is in agreement with different studies on both uniparental and autosomal markers (Dubut et al. 2004; Richard et al. 2007; Ramos-Luis et al. 2014; Karakachoff et al. 2015). However, based on the fineSTRUCTURE results, in our work we detected a stronger evidence of differentiation based on haplotypic data. ADMIXTURE showed a connection to the Irish samples (Supplementary Figure 8), which is also indicated by the ancestry profiles of the B1 and B2 targets, which showed higher proportions for the Irish_Scottish cluster source (Figure 4). The GLOBETROTTER analysis for determining the admixture dates pointed to some interesting results (Supplementary Figure 10). B2, the largest Breton target, gave signals of admixture around $700 \mathrm{CE}$, in the time frame of the British Celtic migrations (from Cornwall and south-west Britain) into Gaulish Armorica (then renamed Brittany) from the 3rd to 9th centuries $\mathrm{CE}$, with a higher flow between the 5th and the 6th centuries CE (Koch 2005). This completely agrees with previous findings (Dubut et al. 2004; Ramos-Luis et al. 2014; Karakachoff et al. 2015). Historical migrations from Ireland to Brittany are well recorded since the 4th century CE (Monnier 1997), as well as the emigration of Irish people during the War of Ireland (1641-1651) into the present day departments of Finistère (FI) and Côte d'Armor (CdA), within which a higher integration of the Irish immigrants is proved by records of marriage, birth and death certificates (Dubut et al. 2004). Furthermore, a Celtic root for the Breton language 
links the Breton departments to the Insular Celtic languages from the British Isles (Forster and Toth 2003).

Still, the connection may be more ancient. In Figure 6, we explore the three main European ancestral components (Lazaridis et al. 2014): the pre-Neolithic huntergatherers, the European Neolithic farmers, and the European Bronze Age steppe. Observing the shared drift with the three hunter-gatherer groups (panels A, B, and C), it is possible to notice how the northwestern departments are mirroring the values shown by the British Isles, the Central-Eastern countries, and Northern Europe. Although it is tempting to speculate that Brittany may be showing a signal of continuity with the British Isles which could be ascribed to a period older than the later Celtic migration, the latter could have also brought this similarity in ancient ancestry profiles. Always Brittany is acting as an outlier in the case of the shared drift with the Steppe Early and Middle Bronze Age group. In Figure 6 (panel E) it is possible to see how Brittany breaks the northeastto-southwest decreasing gradient of shared drift. Even in this context, Brittany shows a continuity with the British Isles. Actually, this is consistent with the archaeological records and the development of a late Megalithic culture that characterized Ireland, Britain and Brittany in a period when other parts of Europe were experiencing the advent of metallurgy (Arias 1999).

\section{Borderlands}

The northeastern rim of France, and the Mediterranean southeastern region represent areas in the perimeter of mainland France that may have received particular genetic influences. In the ancestry profiles (Figure 4), the NE and SE targets exhibit the most complex genetic make-ups, with a diverse array of sources. The Central_Eastern_EU cluster source is mostly represented in the NE target, which includes the departments of Bas-Rhin and Moselle; this area recalls the long history of the Alsace-Lorraine territory: a fuzzy border between France and Germany for a long time, and only recently retroceded to France in 1945 (Blumenthal 2012).

The SE target (most abundant in the Bouches-du-Rhône department) copied from several Mediterranean sources (thus representing the most complex target). This area has been a corridor and a landing place for different Mediterranean peoples, since $600 \mathrm{BCE}$ when 
Greeks established a colony on the Mediterranean coastline of France in the city of Massalia (present-day Marseille) (Fine 1985). However, this Mediterranean connection may have older roots, since the late Epipaelolithic Natufian component (Figure 4), which is found almost exclusively in the Mediterranean populations, is found in France in the highest frequency in the Bouches-du-Rhône department.

\section{Conclusions}

In conclusion, according to our results, France is a genetic intermediate between Central, Eastern, and Northern Europe, with some influences from the Mediterranean countries on the southeastern coast. Analyses with both modern and ancient groups pointed to a clear separation of the southwestern groups (Basques and Gascons) and of Brittany from the rest of the French areas. The application of haplotype-based methods allowed us to look beyond the more homogeneous French haplotypic background, discovering connections with the neighbouring populations (e.g., French northeastern departments with central and eastern Europe), while analyses with ancestral populations strengthened the historical connection between Brittany and the British Isles.

\section{Material and Methods}

\section{Data sources and genotyping}

In this study, informed consent was obtained from 331 individuals from different French departments. Internal Review Board approval for this work was granted by CEICPSMAR ref. 2016/6723/I. The collection of samples (except for Basques and Gascons, see below) was organised in 2007 by Dr. Véronique Van Huffel, from the "Unité de Polymorphisme Génétique" of the "Institut National de la Transfusion Sanguine" in Paris, with the appropriate informed consent. Subsequently, these samples were anonymized and provided to Dr. Eva Ramos-Luis (Universidade de Santiago de Compostela), and were analysed there for Y-chromosome markers (Ramos-Luis et al. 2014) . All the 
subjects and their parents were born in the indicated department in mainland France and bore a French surname. DNA was extracted from blood samples as described in RamosLuis et al. (Ramos-Luis et al. 2014). A total of four Axiom ® Genome-Wide Human Origins Arrays ( 629 K SNPs) (Patterson et al. 2012) were genotyped at the Centro Nacional de Genotipado - Universidade de Santiago de Compostela facility. Genotype calling was performed running four different batches according to the Affymetrix Best Practices Workflow implemented in the software Axiom ${ }^{\mathrm{TM}}$ Analysis Suite 2.0. Out of 331 samples, 52 failed the genotyping process and a total of 279 samples were retained. Three additional samples were removed following an Identity-by-descent analysis (IBD) since they displayed a Proportion IBD value $\geq 0.125$ (minimum threshold for removing relatedness equal or higher than a third degree). Eventually, 276 samples were retained. To complete the French dataset, 79 additional samples from a public source (Lazaridis et al. 2016) and 60 from unpublished data (from an ongoing study on the Basque Country and the Franco-Cantabrian region; samples are subset from those in (Martínez-Cruz et al. 2012)) were added to the original 276 , leading to a total of 415 samples. In a preliminary part of this work, 20 out of the 276 samples were identified as outliers and removed from the study (see Supplementary Figure 1 and caption). Thus, the complete dataset included 256 newly genotyped samples, plus 139 additional ones, for a final group of 395 samples (Dataset A) distributed among 20 different French departments (see Supplementary Figure 2 for the geographical distribution). For the allele frequency analyses, as comparison with external populations, a total of 333 samples were added to Dataset A, forming Dataset B. This external group included 218 samples among Germany, Norway, Spain, Italy, England, Ireland, and Scotland (Lazaridis et al. 2016), together with 107 samples from the Spanish autonomous communities of Catalonia, Valencian Community, and Balearic islands (Biagini et al. 2019), and 8 additional samples from South Italy (Naples) newly genotyped with Axiom ${ }^{\circledR}$ Genome-Wide Human Origins Arrays ( 629 K SNPs). Further 799 samples from external populations (Lazaridis et al. 2016) were added to the previous ones when applying haplotype-based methods (Dataset C). Lastly, in the analysis with ancient data, 282 ancient samples (Lazaridis et al. 2016) were added to the previous dataset, with the only exclusion of the 122 sub-Saharan African samples (Dataset D) since their presence would have reduced the resolution for the distribution of the rest of the samples in the PCA, masking signals of admixture in the dedicated analyses (see Supplementary Figure 3 for the geographical distribution of the modern samples 
from Datasets B and C, and Supplementary table 1 for a summary of the different dataset composition).

\section{Data Quality Control}

Data were prepared using PLINK1.9 (Purcell et al. 2007). Uniparental markers and Xchromosome variants were excluded. For the French dataset, a preliminary set of filters were applied to each group separately before the merging process. We filtered out all variants with missing call rates greater than 5\%, those that failed Hardy-Weinberg test at $\mathrm{p}<10^{-5}$, and samples with more than $10 \%$ missing genotype data. After merging, only variants common to the three datasets were retained and SNPs with a minor allele frequency (MAF) below 5\% were excluded, in order to minimize possible genotyping errors. In the end, 343,884 variants used for haplotype-based methods (Dataset A). For the analyses that needed a set of independent markers (i.e., PCA, Admixture, $F_{S T}$, AMOVA, f3-statistics, EEMS), SNPs were pruned setting a pairwise linkage disequilibrium maximum threshold of 0.5, a window of size 200 and a shift step of 25 . Eventually, the pruned data retained 142,803 variants (Dataset A). In the analyses that included the external populations, only the pruned dataset, consisting in 154,889 SNPs, was used for the allele frequency analyses (Dataset B), while a set of 380,697 variants was retained in the haplotype-based methods (Dataset C). Regarding Dataset D, a set of 163,631 SNPs was retrieved after pruning (See Supplementary table 1 for a summary).

The genotypes of the samples typed for this manuscript can be downloaded from https://figshare.com/articles/France_Dataset/10008689

and https://figshare.com/articles/Naples_Dataset/10008731

\section{Statistical analyses}

PCA

For the principal component analysis, eigenvectors were computed using the SmartPCA program in Eigenstrat software package (v. 13050) (Patterson et al. 2006). For Dataset D, we used the option lsqproject:YES when projecting ancient on top of the modern samples. Results were plotted in R (v 3.0.1). 
$F_{S T}$

The $F_{S T}$ fixation index was computed using the SmartPCA tool (v. 13050) from the Eigenstrat software package. Results were produced in Rstudio (RStudio Team 2015) using $\mathrm{R}$ version 3.4.4 (R Core Team 2018). The $F_{S T}$ matrix was used together with a geographic distance matrix produced with The Geographic Distance Matrix Generator (v. 1.2.3, available from http://biodiversityinformatics.amnh.org/open_source/gdmg) in order to perform a Mantel test correlation using the ade4 (Dray and Dufour 2007) library in R. Results were displayed using ggplot2 (Wickham 2009) and reshape (Wickham 2007) libraries.

\section{AMOVA}

Based on different hierarchical levels (within Departments, Between Departments within Areas/Regions, Between Areas/Regions; see Supplementary Figure 4 for a visual representation of the used Areas and Regions), AMOVA was performed using the poppr.amova function in $\mathrm{R}$ package poppr (v. 2.8.1) (Kamvar et al. 2014, 2015) and significance was tested with the randtest function implemented in $\mathrm{R}$ package ade4. For every percentage of variance, a p-value was calculated based on 1000 permutations.

Admixture

Patterns of population structure were explored, in both Dataset B and D, using ADMIXTURE (Alexander and Novembre 2009) testing from $K=2$ to $K=10$ ancestral clusters and using 10 independent random seeds. Results were represented using the software pong (Behr et al. 2016). For Dataset B, admixture was formally tested with f3 statistics computed using the qp3Pop function implemented in Admixtools (Patterson et al. 2012), while outgroup-f3 statistics were tested for Dataset $D$ in the form of f3(Ancient, X; Mbuti), where 3 Mbuti samples from ref. (Lazaridis et al. 2016) were added to Dataset D (1690 total samples, same variants as in Dataset D).

\section{EEMS (Estimated Effective Migration Surface)}

EEMS (Petkova et al. 2016) analysis was run using Dataset A (142,803 variants from the pruned file). With a matrix of average pairwise genetic dissimilarities calculated using the internal program bed2diffs, a sample coordinates file, and a habitat coordinates file 
generated using Google Earth Pro (v. 7.3.2.5495), we performed 10 pilot runs of 6 million MCMC iterations each, with 3 million burn-in, and a thinning interval of 30,000. A second set of 5 runs was then performed restarting the chain with the highest likelihood with 4 million MCMC iterations, 1 million burn-in, and thinning interval of 10,000. The density of the population grid was set to 300 demes, and random seeds were used for each one of the runs. We used the default hyperparameter values but tuned some of the proposed variances to improve convergence in the second set of runs. Results for the chain with the highest likelihood were displayed using eems.plots function in the $\mathrm{R}$ package rEEMSplots.

\section{Haplotype-based analysis}

Two different analyses were performed: one on the internal French population only (Dataset A), and one also including external populations (Dataset C). In both cases, phasing was performed using the software Shapeit (v. v2.r837) (Delaneau et al. 2014; O’Connell et al. 2014). When running ChromoPainter (Lawson et al. 2012), all samples were used as both recipients and donors, without any population specification (-a option) and not allowing self-copying. First, the parameters for the switch rate and global mutation probability were estimated with the EM algorithm implemented in ChromoPainter using the parameters -i 15 -in -iM for chromosomes 1, 7, 14, and 20 for all the samples. This step allows to estimate the two parameters that will be then averaged for all chromosomes. The outcome for the average weighted values for the global mutation probability and the switch rate parameters were respectively 0.000745 and 266.67196 for Dataset A, and 0.000586 and 237.50784 for Dataset C. In a second step, ChromoPainter was run for all chromosomes using the two fixed parameters. Later, the final coancestry matrices for each chromosome were combined using the tool Chromocombine. The latter also estimates the $\mathrm{C}$ parameter which is needed for the normalization of the coancestry matrix data when we run fineSTRUCTURE in order to identify the population structure. The MCMC of fineSTRUCTURE was run using 1000000 burn-in iterations (flag -x), 2000000 iterations sampled (flag -y), and thinning interval of 10000 (flag -z). Eventually, the fineSTRUCTURE tree was estimated running three different seeds and using the flags $-\mathrm{X}-\mathrm{Y}-\mathrm{m} \mathrm{T}$ that allow to build the sample relationship tree. In the analysis on Dataset $\mathrm{C}$, the work was then divided in two phases. In the first one, ChromoPainter and fineSTRUCTURE were rerun, this time silencing 
France in order to define the external groups only. In the second phase, fineSTRUCTURE was rerun using the "force file" option (-F), using "continents" as donor groups (represented by the external groups defined in the first phase); - $\mathrm{F}$ is a function that allows to exclude the donor representation in the building tree phase and focus on the distribution of the recipient groups, represented by the French samples only. We then applied the nonnegative-least-squares (nnls) function from GLOBETROTTER (Hellenthal et al. 2014) in order to describe the ancestry profiles for the French groups we detected with the "force file" option. We then used GLOBETROTTER in order to describe admixture events, sources and dates. More details about the usage of GLOBETROTTER are reported in Supplementary note 1 .

\section{ACKNOWLEDGMENTS}

We thank Dr. Véronique Van Huffel, from the "Unité de Polymorphisme Génétique" of the "Institut National de la Transfusion Sanguine" in Paris for collecting most of the samples used in this paper, and Inés Quintela and the National Genotyping Center (CEGEN - USC) for their assistance in genotyping the samples. Of course, this work would not have been possible without the kind collaboration of all the sample donors. Funding was provided by the Agencia Estatal de Investigación and Fondo Europeo de Desarollo Regional (FEDER) (grant CGL2016-75389-P), Agència de Gestió d'Ajuts Universitaris i de la Recerca (Generalitat de Catalunya) grant 2014 SGR 866, and "Unidad de Excelencia María de Maeztu”, funded by the MINECO (ref: MDM-20140370). SAB was supported by the Agencia Estatal de Investigación FPI grant BES-2014069224.

\section{Conflicts of interest}

On behalf of all authors, the corresponding author states that there is no conflict of interest.

\section{References}

1000 Genomes Project Consortium, Auton A, Brooks LD, et al (2015) A global reference for human genetic variation. Nature 526:68-74. https://doi.org/10.1038/nature15393 
Alexander DH, Novembre J (2009) Fast Model-Based Estimation of Ancestry in Unrelated Individuals. Genome Res 19:1655-1664. https://doi.org/10.1101/gr.094052.109.vidual

Arias P (1999) The Origins of the Neolithic Along the Atlantic Coast of Continental Europe: A Survey. J World Prehistory 13:403-464

Behr AA, Liu KZ, Liu-Fang G, et al (2016) pong: fast analysis and visualization of latent clusters in population genetic data. Bioinformatics 32:2817-2823. https://doi.org/10.1093/bioinformatics/btw327

Biagini SA, Solé-Morata N, Matisoo-Smith E, et al (2019) People from Ibiza: an unexpected isolate in the Western Mediterranean. Eur J Hum Genet 27:941-951. https://doi.org/10.1038/s41431-019-0361-1

Blumenthal D (2012) Alsace-Lorraine: A Study of the Relations of the Two Provinces to France and to Germany, and a Presentation of the Just Claims of Their People (Classic Reprint). Forgotten Books, London

Bycroft C, Fernandez-Rozadilla C, Ruiz-Ponte C, et al (2019) Patterns of genetic differentiation and the footprints of historical migrations in the Iberian Peninsula. Nat Commun 10:551. https://doi.org/10.1038/s41467-018-08272-w

Calafell F, Bertranpetit J (1994) Principal component analysis of gene frequencies and the origin of Basques. Am J Phys Anthropol 93:201-215. https://doi.org/10.1002/ajpa.1330930205

Calafell F, Bertranpetit J (1993) A simulation of the genetic history of the Iberian Peninsula. Curr Anthropol 34:735-745

Cambon-Thomsen A, Ohayon E (1988) Practical Application of Population Genetics: The Genetic Survey "Provinces Françaises." In: Mayr WR (ed) Advances in Forensic Haemogenetics. Advances in Forensic Haemogenetics, vol 2. pp 535-553

Delaneau O, Marchini J, McVeanh GA, et al (2014) Integrating sequence and array data to create an improved 1000 Genomes Project haplotype reference panel. Nat Commun 5:1-9. https://doi.org/10.1038/ncomms4934

Dray S, Dufour A-B (2007) The ade4 Package: Implementing the Duality Diagram for Ecologists. J Stat Softw 22: https://doi.org/10.18637/jss.v022.i04

Dubut V, Chollet L, Murail P, et al (2004) mtDNA polymorphisms in five French groups: importance of regional sampling. Eur J Hum Genet 12:293-300. https://doi.org/10.1038/sj.ejhg.5201145

Fine JVA (1985) The Ancient Greeks: A Critical History. Belknap Press: An Imprint of 
Harvard University Press

Forstenzer TR (2016) French Provincial Police and the Fall of the Second Republic: Social Fear and Counterrevolution. Princeton University Press, Princeton, NJ

Forster P, Toth A (2003) Toward a phylogenetic chronology of ancient Gaulish, Celtic, and Indo-European. Proc Natl Acad Sci 100:9079-9084. https://doi.org/10.1073/pnas.1331158100

Günther T, Valdiosera C, Malmström H, et al (2015) Ancient genomes link early farmers from Atapuerca in Spain to modern-day Basques. Proc Natl Acad Sci U S A 112:11917-22. https://doi.org/10.1073/pnas.1509851112

Haine WS (2000) The history of France. Greenwood Press, Santa Barbara, CA Hellenthal G (2015) Instruction Manual for “ GLOBETROTTER : a program for identifying, dating and describing admixture events in population data " for GLOBETROTTER. 1-24

Hellenthal G, Busby GBJ, Band G, et al (2014) A genetic atlas of human admixture history. Science (80- ) 343:747-751. https://doi.org/10.1126/science.1243518

House GL, Hahn MW (2018) Evaluating methods to visualize patterns of genetic differentiation on a landscape. Mol Ecol Resour 18:448-460. https://doi.org/10.1111/1755-0998.12747

Kamvar ZN, Brooks JC, Grünwald NJ (2015) Novel R tools for analysis of genomewide population genetic data with emphasis on clonality. Front Genet 6:. https://doi.org/10.3389/fgene.2015.00208

Kamvar ZN, Tabima JF, Grünwald NJ (2014) Poppr : an R package for genetic analysis of populations with clonal, partially clonal, and/or sexual reproduction. PeerJ 2:e281. https://doi.org/10.7717/peerj.281

Karakachoff M, Duforet-Frebourg N, Simonet F, et al (2015) Fine-scale human genetic structure in Western France. Eur J Hum Genet 23:831-836. https://doi.org/10.1038/ejhg.2014.175

Kherumian R, Moullec J, Nguyen VC (1967) Groupes sanguins érythrocytaires A, A2, $\mathrm{BO}, \mathrm{MN}, \mathrm{Rh}(\mathrm{CcDE})$ et sériques, $\mathrm{Hp}, \mathrm{Tf}, \mathrm{Gm}$ dans quatre régions militaires françaises. Bull Mem Soc Anthropol Paris 1:377-384. https://doi.org/10.3406/bmsap.1967.1396

Koch J (2005) Breton Migrations. In: Celtic Culture : A Historical Encyclopedia. ABCCLIO, Santa Barbara, CA, pp 275-277

Lao O, Lu TT, Nothnagel M, et al (2008) Correlation between Genetic and Geographic 
Structure in Europe. Curr Biol 18:1241-1248

Lawson DJ, Hellenthal G, Myers S, Falush D (2012) Inference of Population Structure using Dense Haplotype Data. PLoS Genet 8:e1002453.

https://doi.org/10.1371/journal.pgen.1002453

Lazaridis I, Nadel D, Rollefson G, et al (2016) Genomic insights into the origin of farming in the ancient Near East. Nature 536:419-424. https://doi.org/10.1038/nature19310

Lazaridis I, Patterson N, Mittnik A, et al (2014) Ancient human genomes suggest three ancestral populations for present-day Europeans. Nature 513:409-413. https://doi.org/10.1038/nature13673

Leslie S, Winney B, Hellenthal G, et al (2015) The fine-scale genetic structure of the British population. Nature 519:309-314. https://doi.org/10.1038/nature14230

MacMaster N (1997) Colonial Migrants and Racism Algerians in France, 1900-62. Palgrave Macmillan

Martínez-Cruz B, Harmant C, Platt DE, et al (2012) Evidence of pre-Roman tribal genetic structure in Basques from uniparentally inherited markers. Mol Biol Evol 29:2211-22. https://doi.org/10.1093/molbev/mss091

Monnier J (1997) Chapitre 6 : L'immigration bretonne en Armorique. In: Monnier J, Cassard J (eds) Toute l'histoire de Bretagne. Skol Vreizh, pp 97-106

Novembre J, Johnson T, Bryc K, et al (2008) Genes mirror geography within Europe. Nature 456:98-101

O’Connell J, Gurdasani D, Delaneau O, et al (2014) A General Approach for Haplotype Phasing across the Full Spectrum of Relatedness. PLoS Genet 10:e1004234. https://doi.org/10.1371/journal.pgen.1004234

OECD (2017) OECD Multi-level Governance Studies Multi-level Governance Reforms Overview of OECD Country Experiences

Olalde I, Mallick S, Patterson N, et al (2019) The genomic history of the Iberian Peninsula over the past 8000 years. Science 363:1230-1234. https://doi.org/10.1126/science.aav4040

Patterson N, Moorjani P, Luo Y, et al (2012) Ancient Admixture in Human History. Genetics 192:1065-1093. https://doi.org/10.1534/genetics.112.145037

Patterson N, Price AL, Reich D (2006) Population structure and eigenanalysis. PLoS Genet 2:2074-2093. https://doi.org/10.1371/journal.pgen.0020190

Pereira L, Richards M, Goios A, et al (2005) High-resolution mtDNA evidence for the 
late-glacial resettlement of Europe from an Iberian refugium. Genome Res 15:1924. https://doi.org/10.1101/gr.3182305

Petkova D, Novembre J, Stephens M (2016) Visualizing spatial population structure with estimated effective migration surfaces. Nat Genet 48:94-100. https://doi.org/10.1038/ng.3464

Purcell S, Neale B, Todd-Brown K, et al (2007) PLINK: a tool set for whole-genome association and population-based linkage analyses. Am J Hum Genet 81:559-75. https://doi.org/10.1086/519795

R Core Team (2018) R: A Language and Environment for Statistical Computing. R Foundation for Statistical Computing, Vienna.

Ramos-Luis E, Blanco-Verea A, Brión M, et al (2014) Y-chromosomal DNA analysis in French male lineages. Forensic Sci Int Genet 9:162-168. https://doi.org/10.1016/j.fsigen.2013.12.008

Ramos-Luis E, Blanco-Verea A, Brión M, et al (2009) Phylogeography of French male lineages. Forensic Sci Int Genet Suppl Ser 2:439-441

Raveane A, Aneli S, Montinaro F, et al (2019) Population structure of modern-day Italians reveals patterns of ancient and archaic ancestries in Southern Europe. Sci Adv 5:1-13. https://doi.org/10.1126/sciadv.aaw3492

Richard C, Richard C, Pennarun E, et al (2007) An mtDNA perspective of French genetic variation. Ann Hum Biol 34:68-79. https://doi.org/10.1080/03014460601076098

Rodríguez-Ezpeleta N, Álvarez-Busto J, Imaz L, et al (2010) High-density SNP genotyping detects homogeneity of Spanish and French Basques, and confirms their genomic distinctiveness from other European populations. Hum Genet 128:113-117. https://doi.org/10.1007/s00439-010-0833-4

RStudio Team (2015) RStudio Team (2015). RStudio: Integrated Development for R. Sowerwine C (2009) France since 1870: Culture, Society and the Making of the Republic, 2nd edn. Palgrave Macmillan

Tian C, Kosoy R, Nassir R, et al (2009) European population genetic substructure: Further definition of ancestry informative markers for distinguishing among diverse European ethnic groups. Mol Med 15:371-383. https://doi.org/10.2119/molmed.2009.00094

Wickham H (2009) ggplot2. Springer New York, New York, NY Wickham H (2007) Reshaping Data with the reshape Package. J Stat Softw 21: 
https://doi.org/10.18637/jss.v021.i12

Zuazo K (2010) El euskera y sus dialectos. Alberdania, Zarautz (Gipuzkoa) 


\section{Main Figures and Tables}

\begin{tabular}{|c|c|c|c|}
\hline Groupings & $\%$ Total variance & $\Phi$-statistics & $\mathrm{p}$ \\
\hline Variations Between Areas & 0.02 & $\Phi_{\mathrm{ST}}=0.0026$ & 0.4605 \\
\hline Variations Between Departments Within Areas & 0.23 & $\Phi_{\mathrm{ST}}=0.0023$ & 0.0009 \\
\hline Variations Within Departments & 99.73 & $\Phi_{\mathrm{ST}}=0.00027$ & 0.0009 \\
\hline Variations Between Regions & -0.054 & $\Phi_{S T}=-0.0005$ & 0.7362 \\
\hline Variations Between Departments Within Regions & 0.3 & $\Phi_{\mathrm{ST}}=0.003$ & 0.0009 \\
\hline Variations Within Departments & 99.74 & $\Phi_{\mathrm{ST}}=0.0025$ & 0.0009 \\
\hline Variations Between Departments & 0.26 & $\Phi_{\mathrm{ST}}=0.0026$ & 0.0009 \\
\hline Variations Within Departments & 99.73 & & \\
\hline
\end{tabular}

Table 1. Hierarchical analysis of molecular variance (AMOVA). Results for percentage of total variance, $\Phi$-statistics, and p-values are reported for the three distinct analyses. A) proportion of genetic variation partitioned among geographic areas, among departments within geographic areas, and within departments; B) proportion of genetic variation partitioned among regions, among departments within regions, and within departments; C) proportion of genetic variation partitioned among departments and within departments. Regions refer to the division established in 2016, areas are defined based on the geographic distribution of departments. 


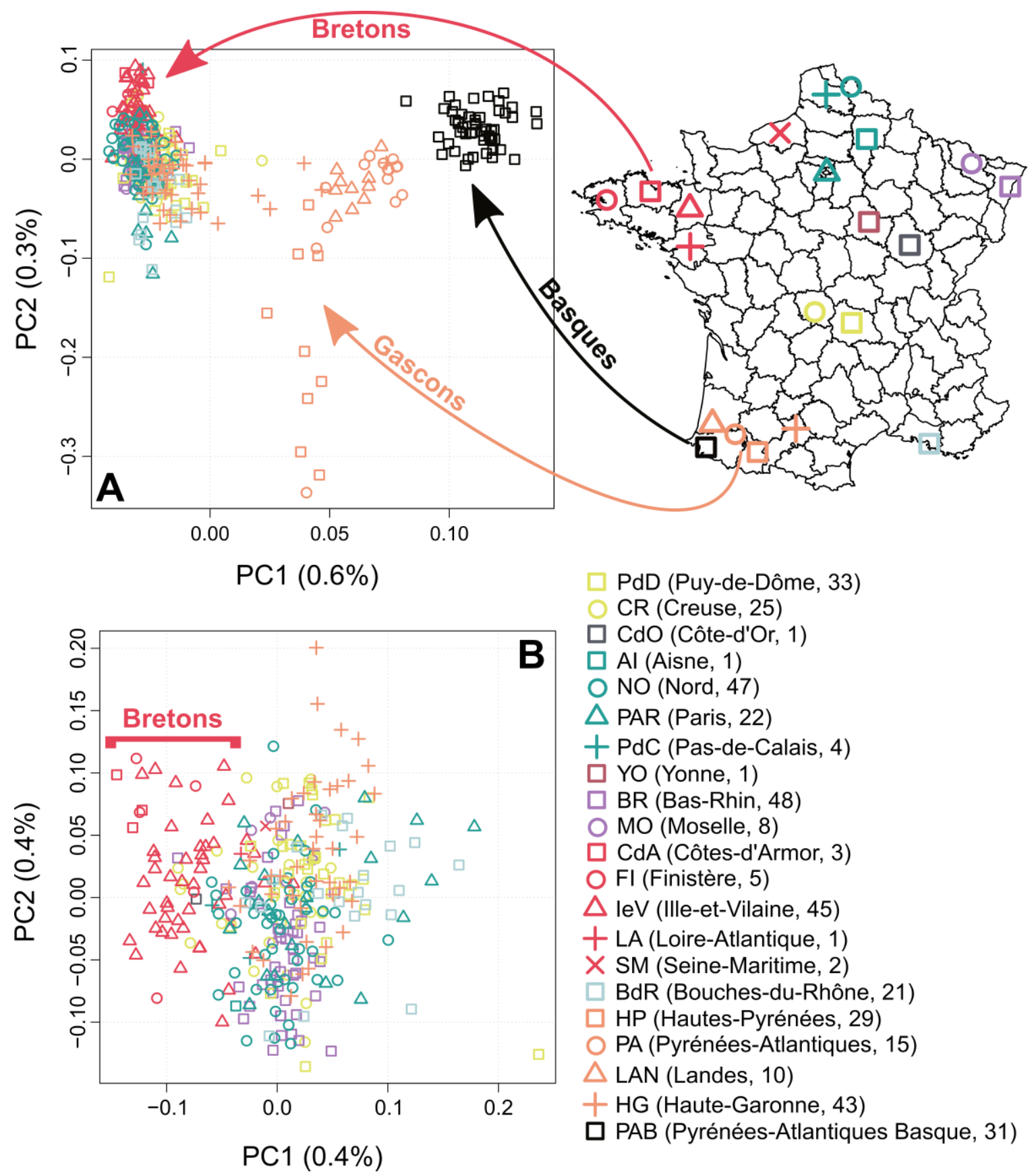

Figure 1. Principal Component Analysis of French samples (dataset A) with A) Basque and Gascon samples, and B) without them. Colors correspond to distinct geographic areas, while different symbols with the same color represent distinct departments in each area (See map distribution). Only Basques are colored differently to stress the difference from the non-Basque-speaking samples from that same area. Legend displays acronyms as used in the main text. The extended department names are reported in parenthesis together with the corresponding sample size. 


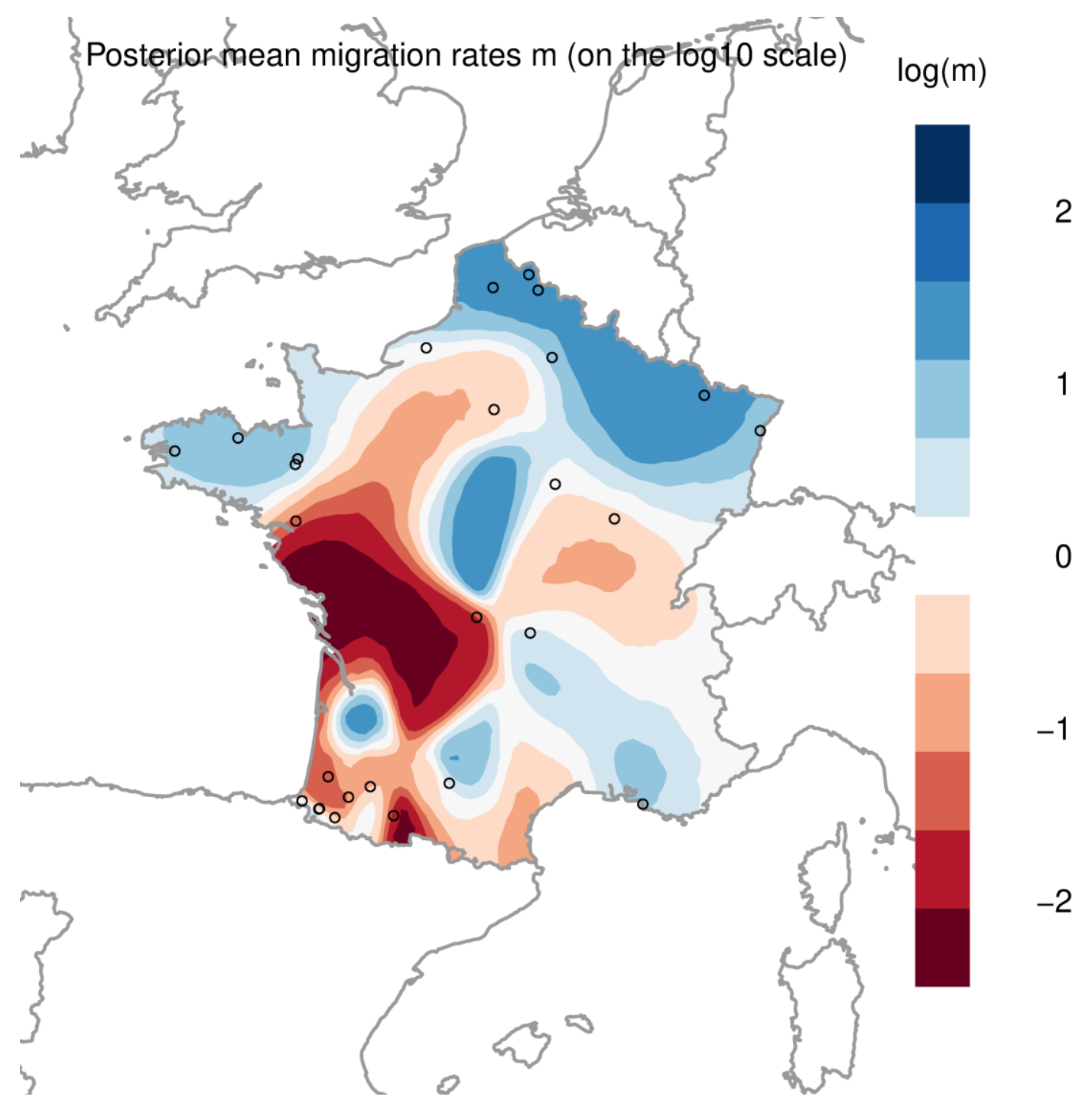

Figure 2. EEMS plot based on 395 French samples (Dataset A). Different shades of the same color represent differential levels of high (blue) or low (red) effective migration rates. The zero value indicates the average effective migration rate. Geographical locations for the different departments are averages of the coordinates among samples. 

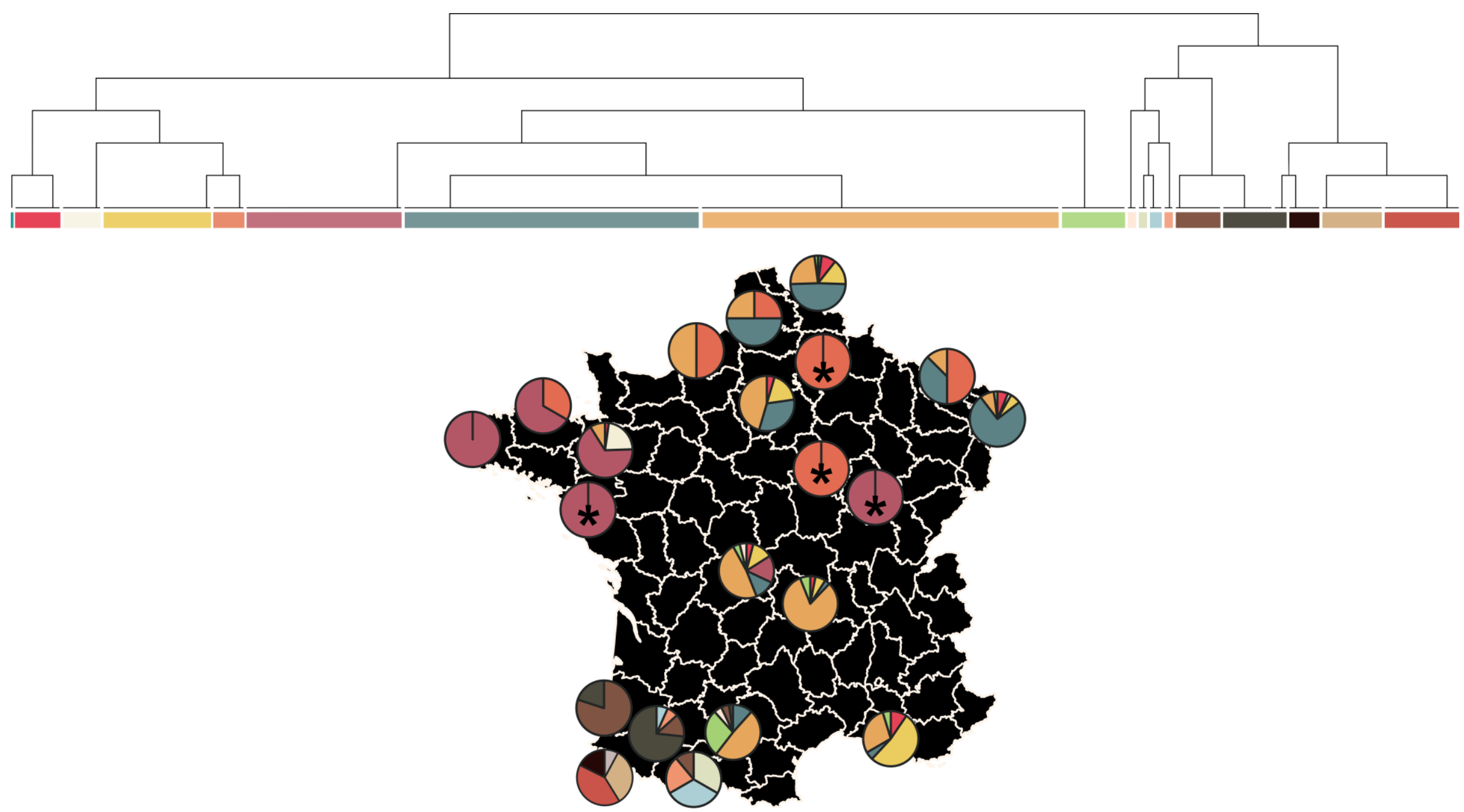

Figure 3. Pie charts showing the spatial distribution of haplotypes inferred by the fineSTRUCTURE tree. Each pie chart is a department, while colors correspond to the clusters described in the tree above the map. See Figure 1 for department names. Asterisks indicate departments with only one sample. 


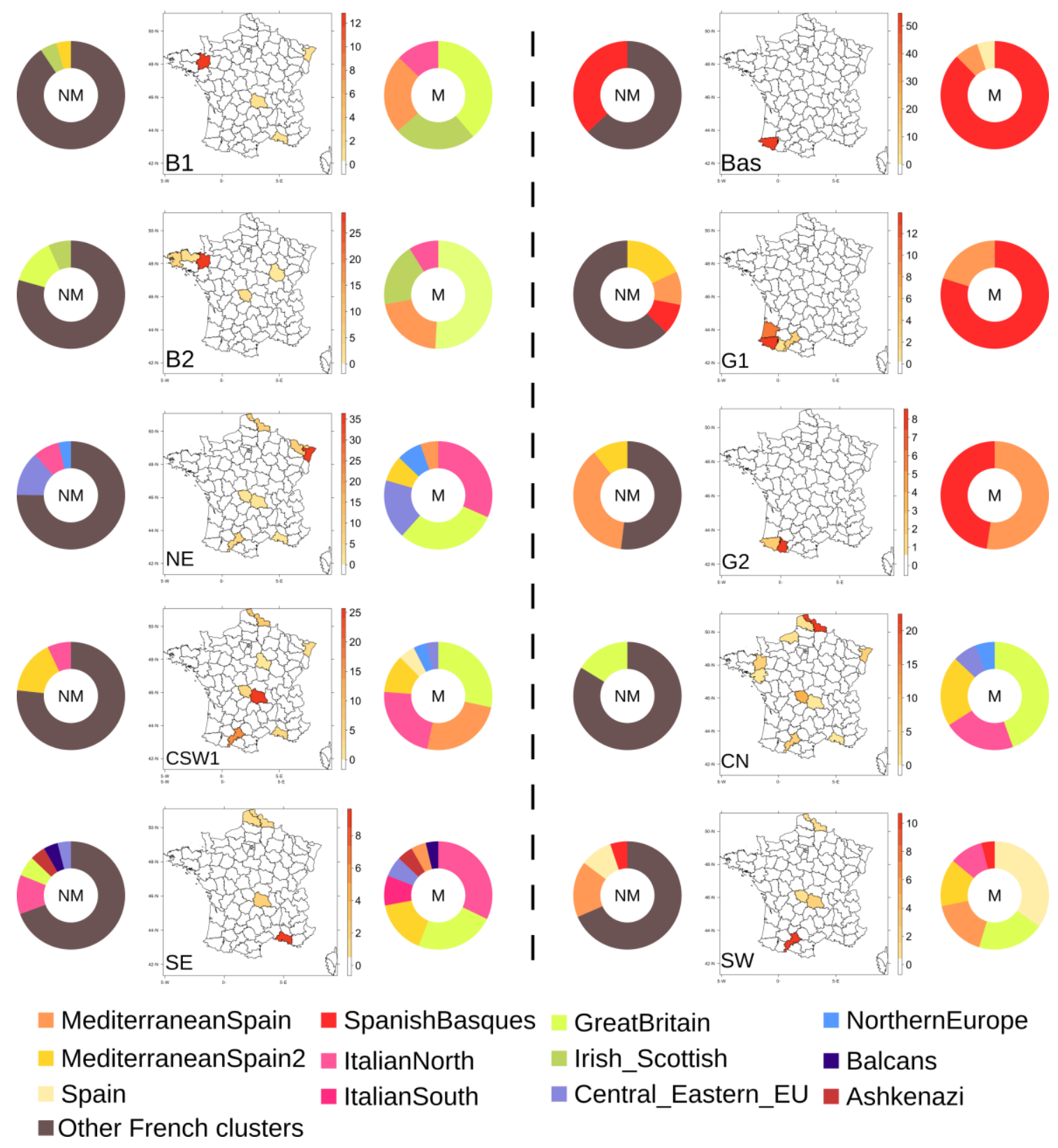

Figure 4. Ancestry profiles for 10 French targets. Each map is a target defining a specific major area of the French territory. On the left of each map, the donut chart is representing the ancestry profile for the not masked analysis (NM); on the right the same analysis has been masked (M). The different colors represent proportions of haplotype sharing with a specific source (only contributions above the $2.5 \%$ are shown); sources are defined in supplementary Figure 9. In the NM analysis, the brown color refers to contributions coming from other French groups (cumulative value). Target names stand for: B1 and B2, Brittany; NE, NorthEast; CSW1, Central-SouthWest; SE, SouthWest; Bas, Basques; G1 and G2, Gascons; CN, CentralNorth; SW, SouthWest 


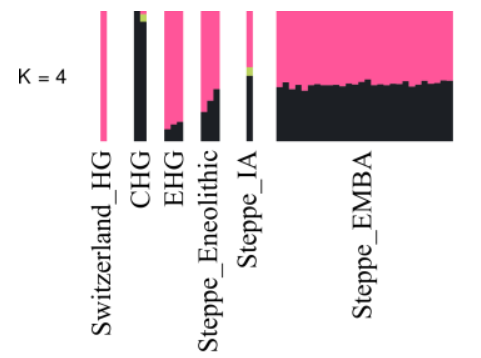

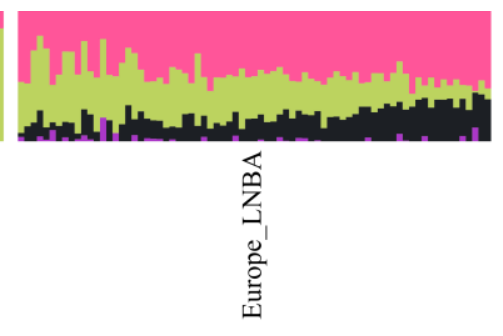

Central Europe

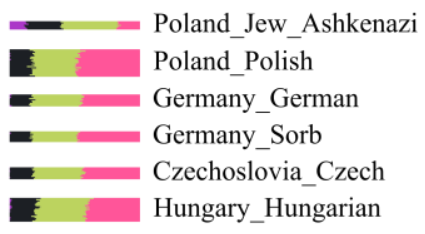

Southeastern Europe

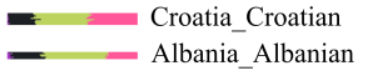

_ Albania_Albanian

- Romania Romania

Greece_Greek

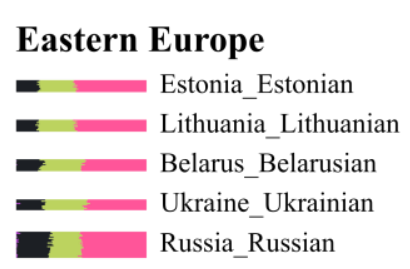

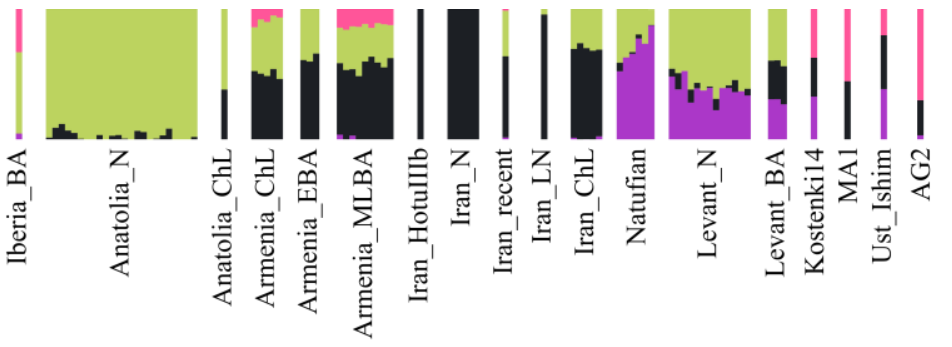

\section{British Isles}

E Ireland_Irish

- Ireland_Irish_Ulster

- UK_English

I UK_Scottish

- UK_Shetlandic

- OrkneyIslands_Orcadia

\section{Scandinavia}

= Iceland_Icelandic

- Norway_Norwegian

- Finland_Finnish

\section{Caucasus}

- S Spi__biza.

Spain_Valencian_Community

Italy_Italian_North

_ Italy_Italian_South

- _ Italy_Italian_South_Naples

Italy_Sardinian

- Italy_Sicily

_ $\quad$ Malta_Maltese
Central/Western Asia

Lebanon_Lebanese

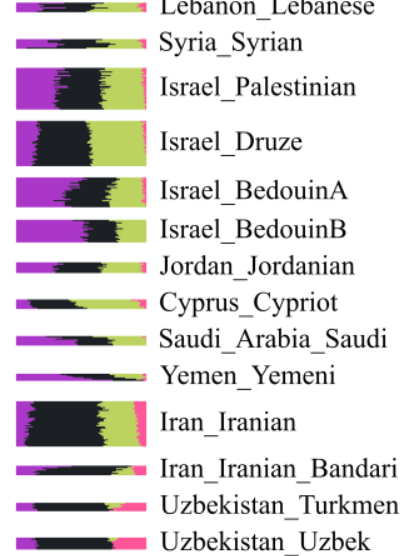

Northern Africa

$=$ Morocco_Moroccan - Algeria Algerian Algeria_Mozabite

$=$ Tunisia_Tunis

2.1Eypt_Egyptian
- Tunisia_Tunisian

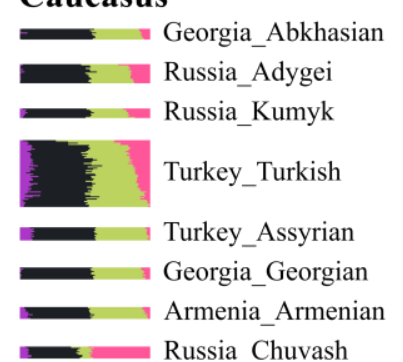

Figure 5. ADMIXTURE results for K=4 ancestral components using dataset D. Results for the ancient samples are on the top of the figure. Below, modern samples are organized according to major geographical groupings. 


\section{A. $\mathbf{f} 3$ (WHG,X;Mbuti)}

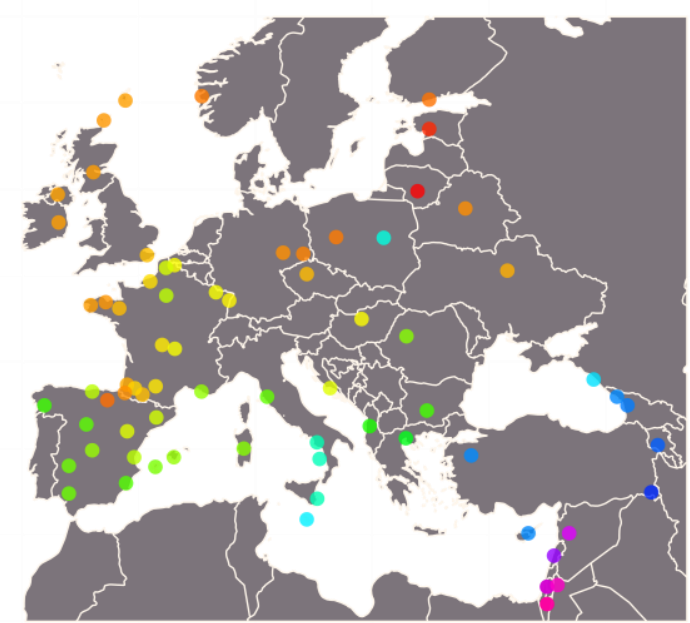

D. f3(Europe_EN,X;Mbuti)

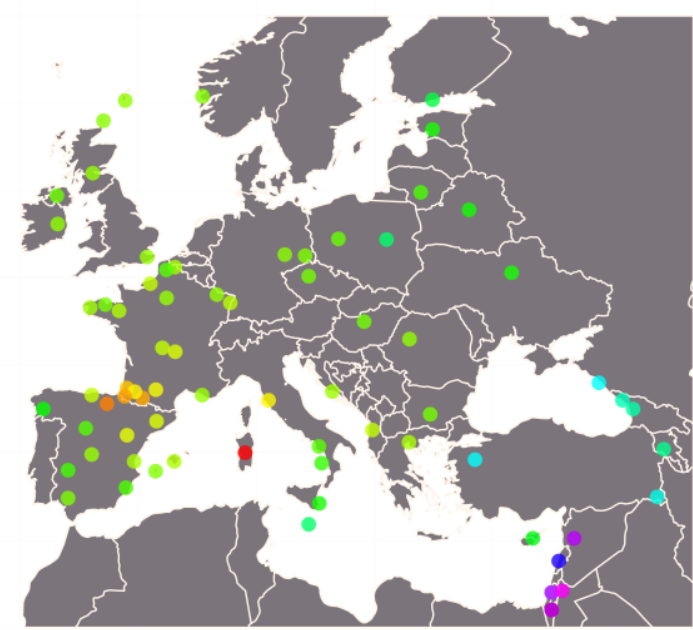

\section{B. $f 3(E H G, X ; M b u t i)$}

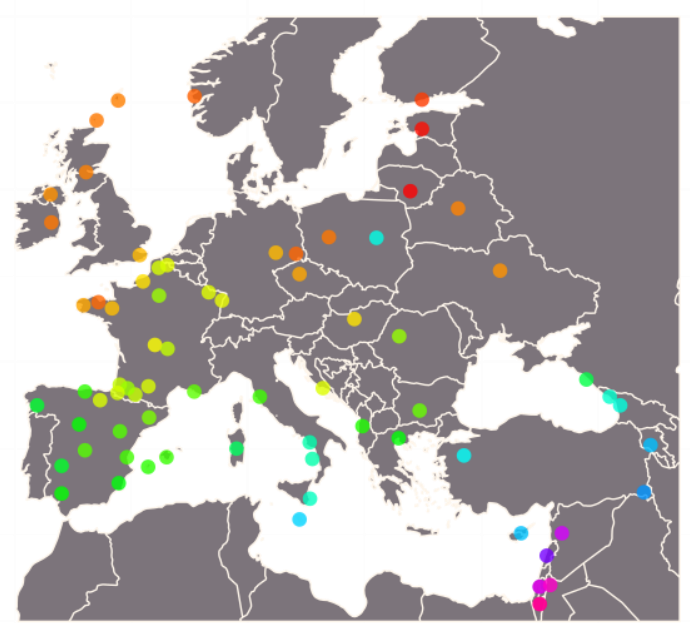

E. f3(Steppe_EMBA,X;Mbuti)
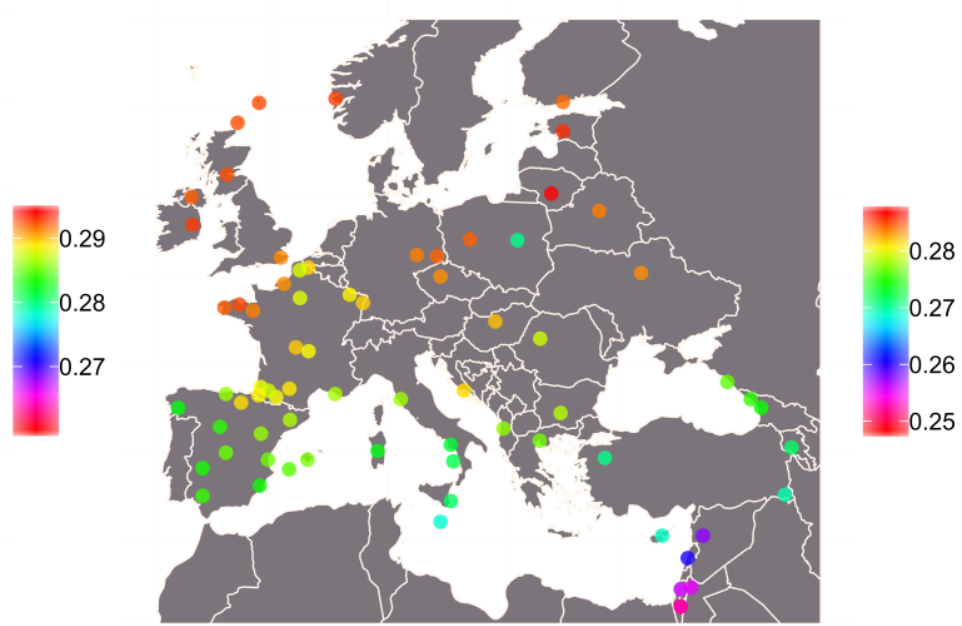

\section{C. f3(SHG,X;Mbuti)}

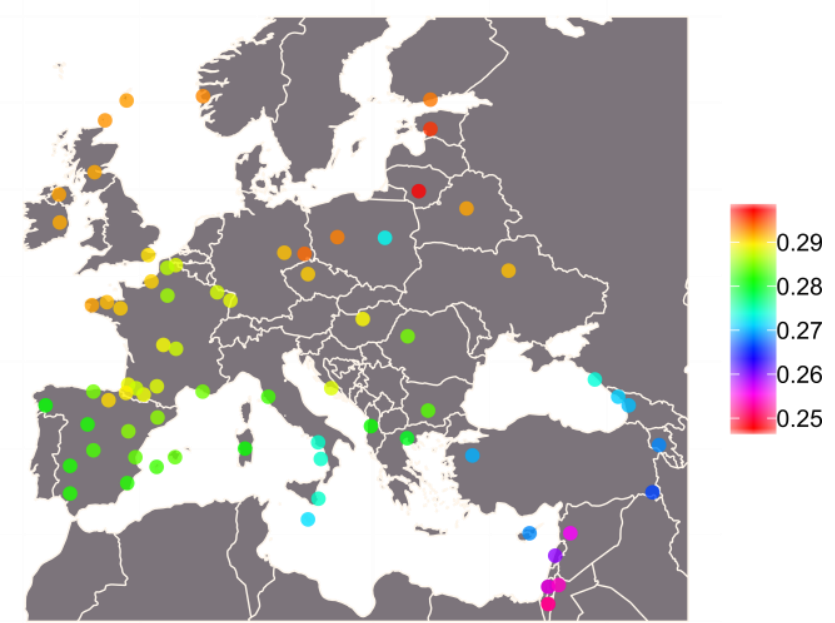

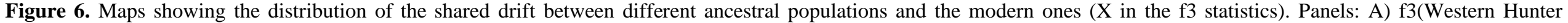

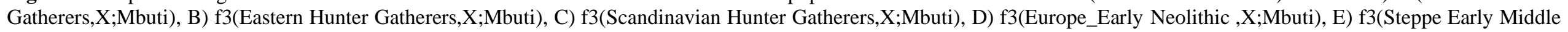
Bronze Age,X;Mbuti). In France, departments with less than two individuals are not shown. 


\section{Supplementary Data}

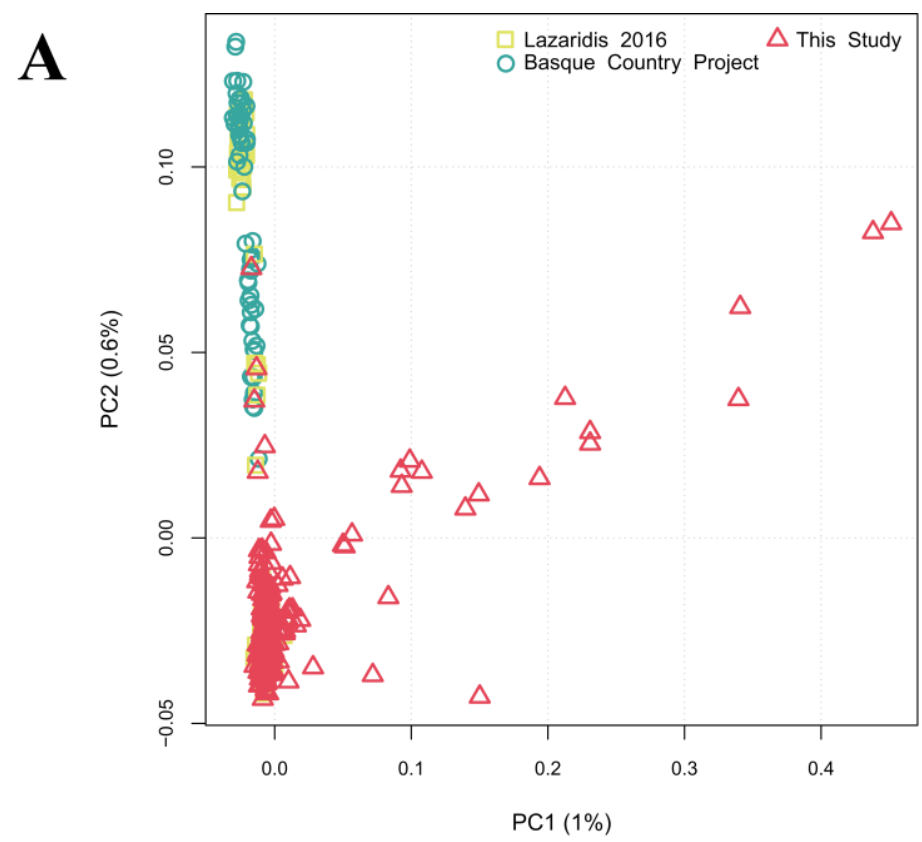

B
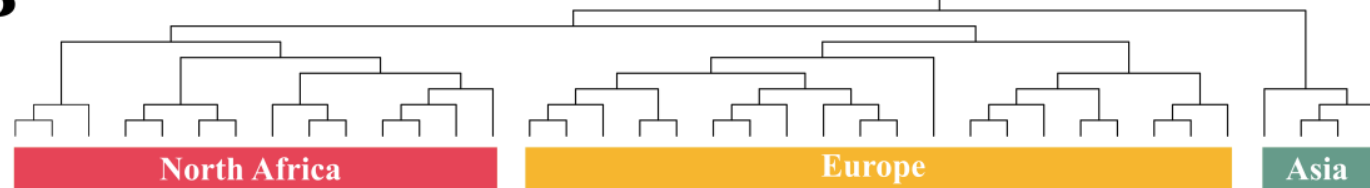

Supplementary Figure 1. PCA with 415 French samples highlighted the presence of outliers clearly skewing the global distribution of the samples (A). We assessed the origin of those samples using ChromoPainter and fineSTRUCTURE in the context of external references from three worldwide populations (CEU, YRI, CHB) from the 1000 genomes project (1nd North African samples from published data (L Four clusters were defined (B), assigning the majority of our samples (395) to the European cluster. The remaining 20 were outliers mainly belonging to the North African cluster (16 samples), 2 samples each were instead assigned to the Asian and the Sub-Saharan African clusters. 


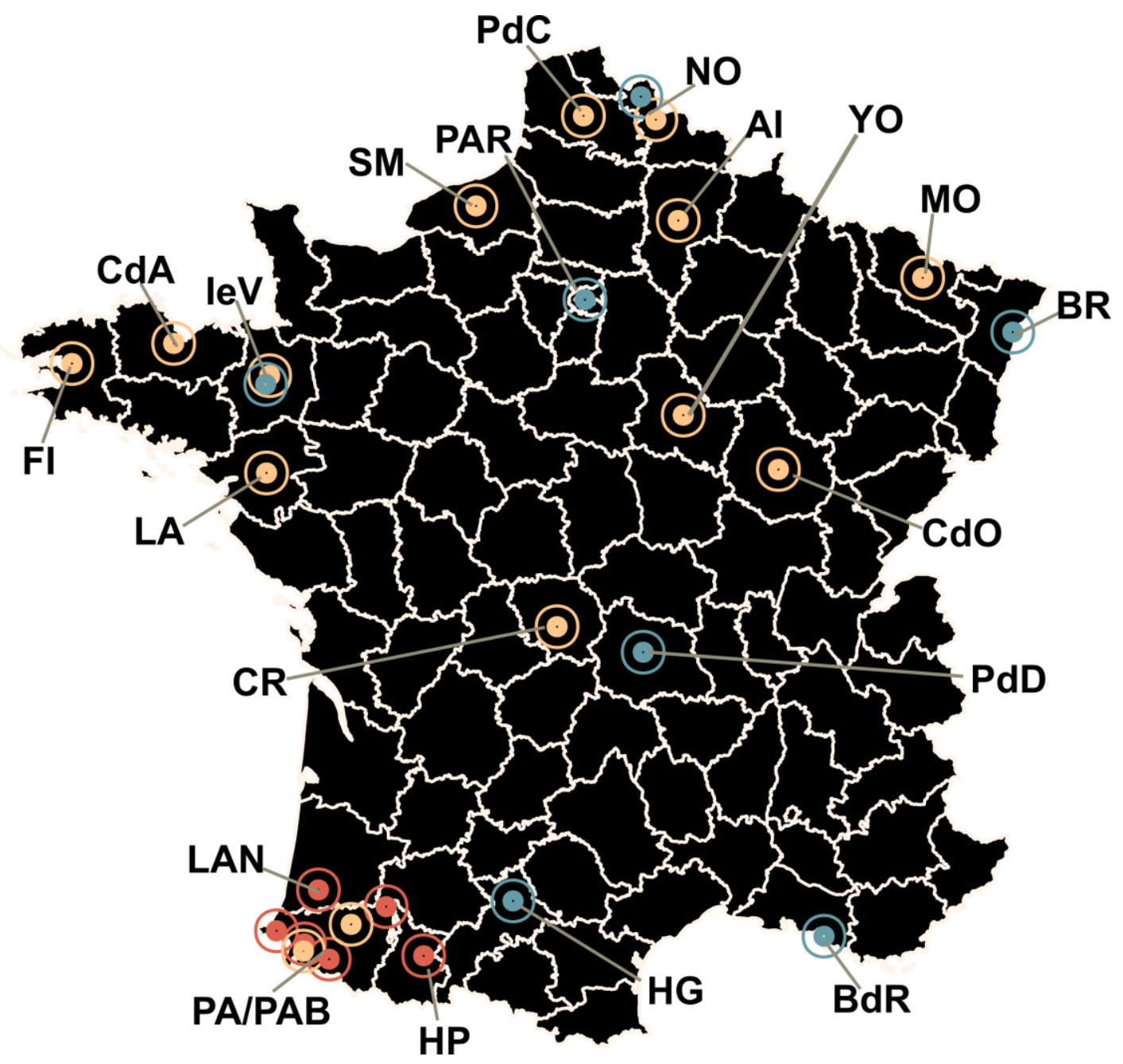

Supplementary Figure 2. Map showing sample distribution among the different departments. Geographical coordinates are averages among samples. Different colors define the three datasets used in this work (blue dots correspond to the 256 samples genotyped for this work; yellow dots correspond to the 79 samples from Lazaridis et al., 2016; red dots correspond to the 60 samples from unpublished data). Sample size and acronyms for the departments are: PdD, Puy-de-Dôme (33); CR, Creuse (25); CdO, Côted'Or (1); AI, Aisne (1); NO, Nord (47); PdC, Pas-de-Calais (4); PAR, Paris (22); YO, Yonne (1); MO, Moselle (8); BR, Bas-Rhin (48); IeV, Ille-et-Vilaine (45); CdA, Côtes-d'Armor (3); FI, Finistère (5); SM, Seine-Maritime (2); LA, Loire-Atlantique (1); BdR, Bouches-du-Rhône (21); LAN, Landes (10); HG, Haute-Garonne (43); PA, Pyrénées-Atlantiques (15); PAB, Pyrénées-Atlantiques Basque (31); HP, HautesPyrénées (29). 


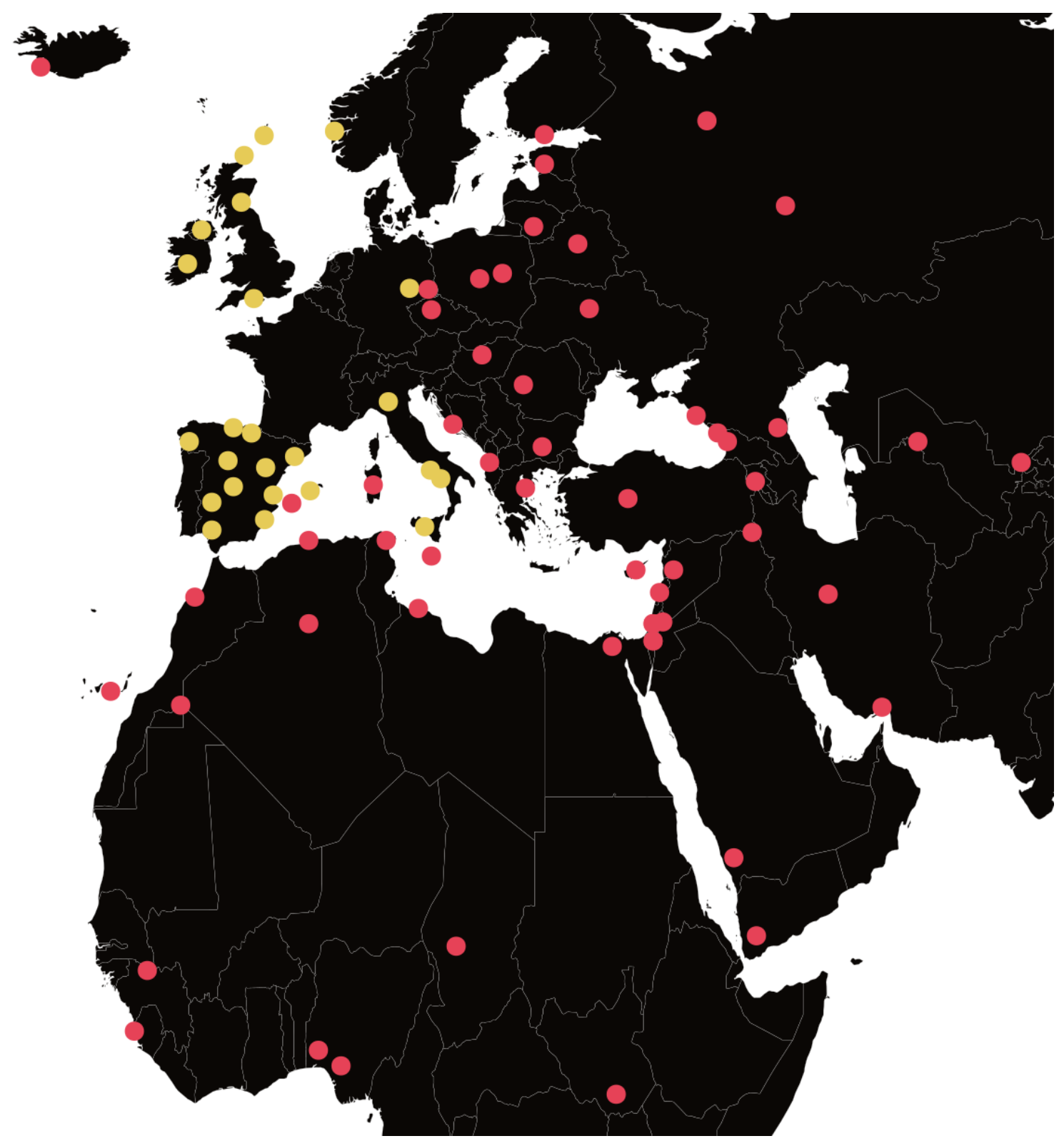

Supplementary Figure 3. External group distribution. Average geolocation points for the 79 external populations are displayed. Yellow dots refer to the 333 samples included in the allele frequency analyses. Yellow and red points together represent the 1132 samples used in the haplotype-based analyses 

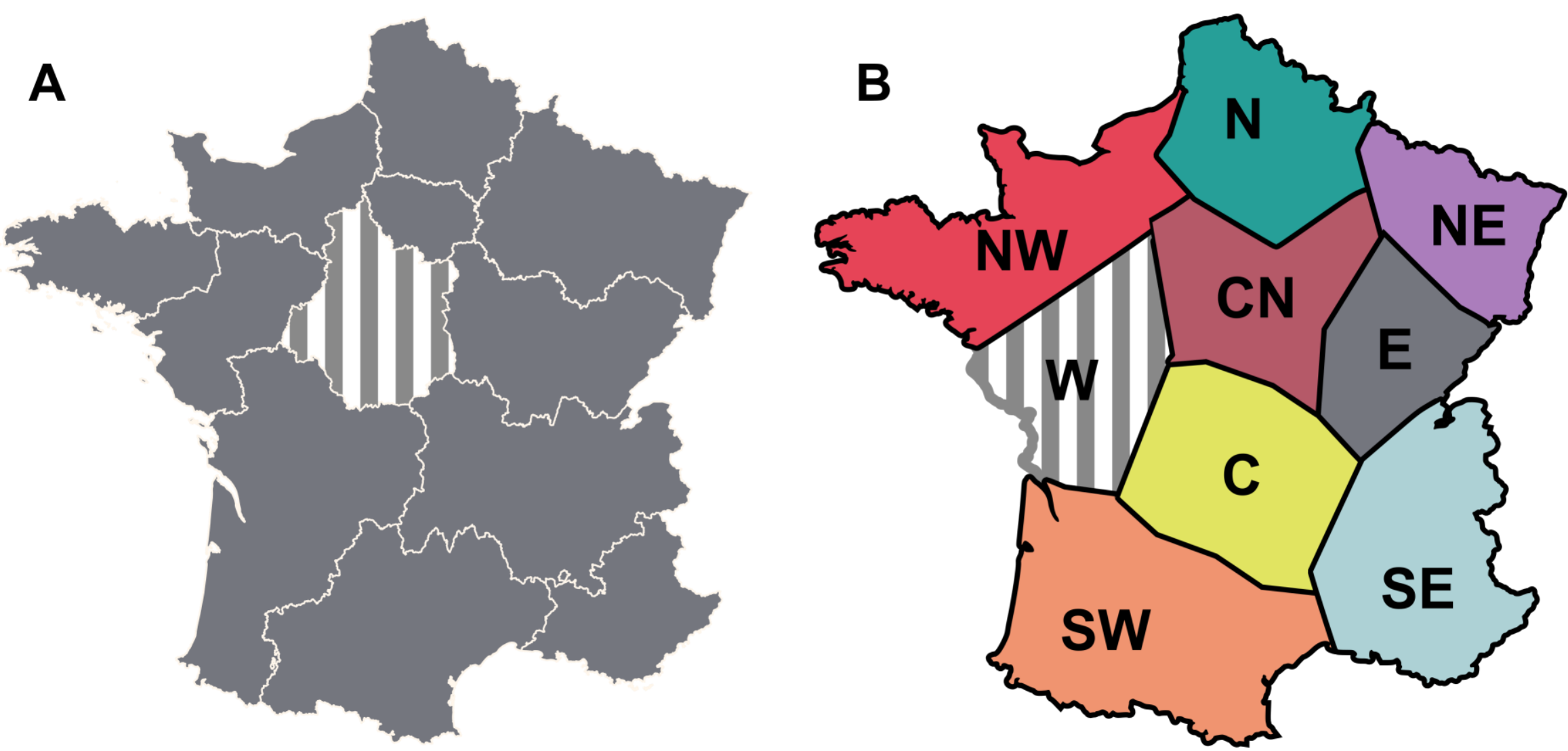

Supplementary Figure 4. Higher hierarchical levels used in the AMOVA analysis for A) Regions and B) Areas. Grey vertical lines highlight unsampled zones. Acronyms for the Areas are: NW, Northwest; N, North; NE, Northeast; W, West; CN, Central North; E, East; C, Center; SW, Southwest; SE, Southeast. 

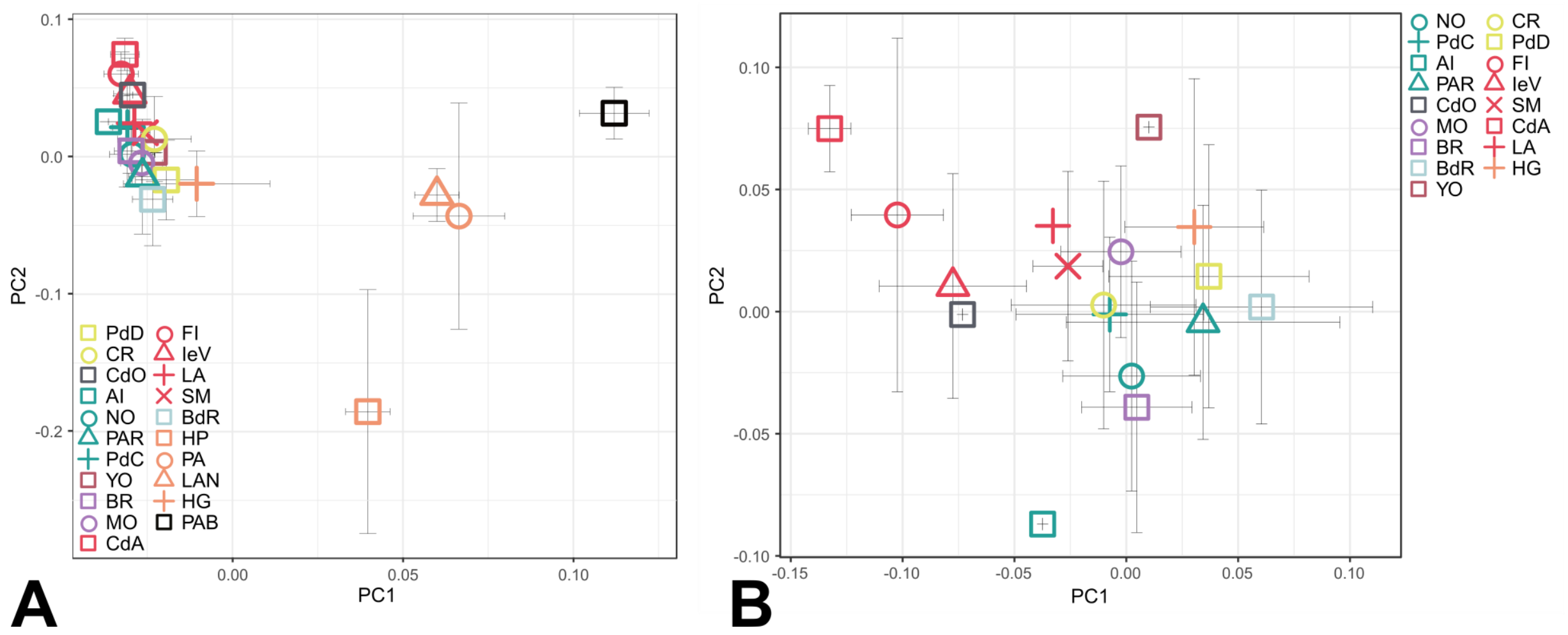

Supplementary Figure 5. Averaged Principal Component Analysis with A) Basque and Gascon samples, and B) without them. Color and symbol codes are the same as in main Figure 2. For each group, each averaged eigenvalue is represented along with standard deviation bars for the two PCs. 

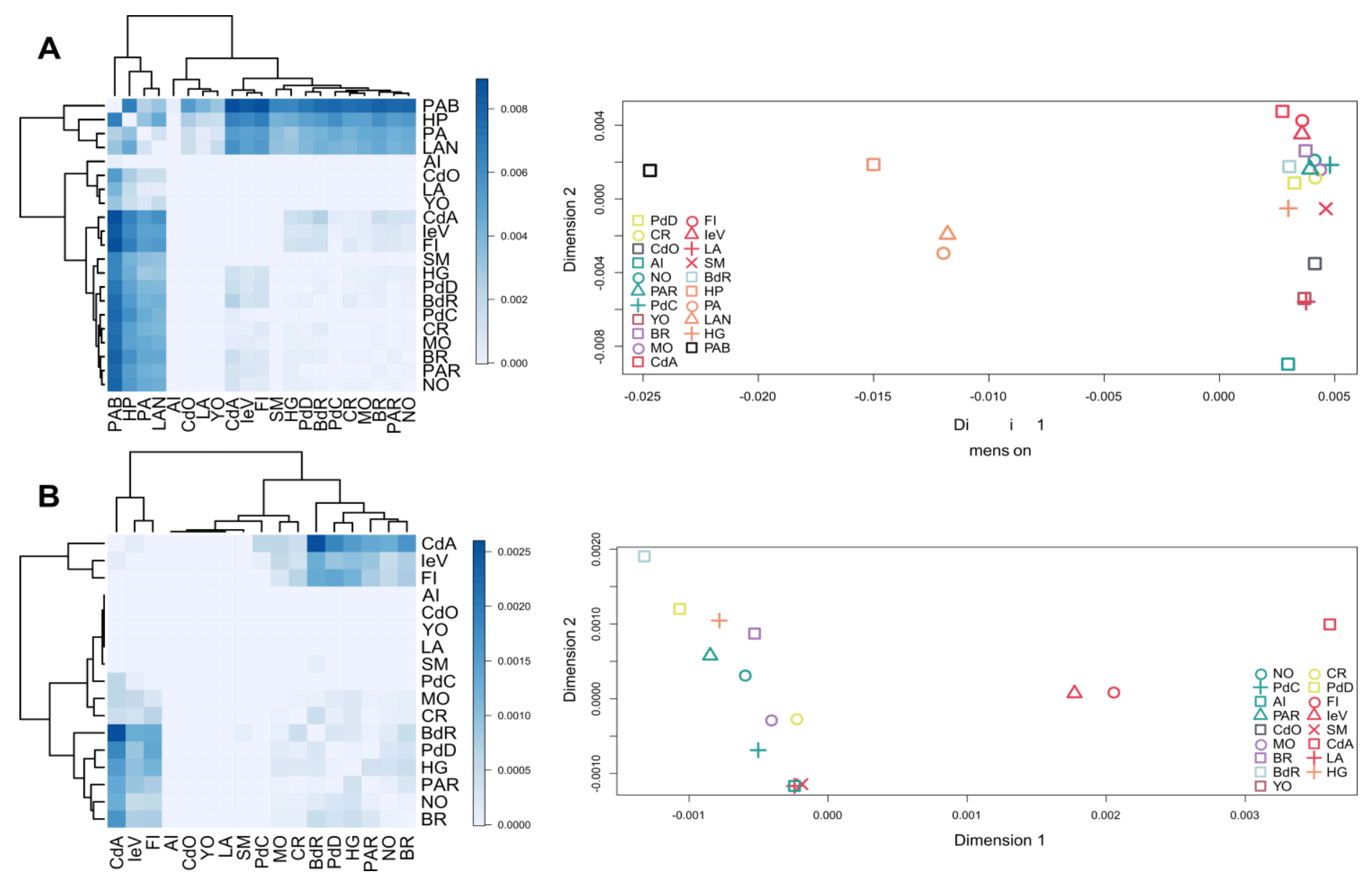

Supplementary Figure 6. On the left: heatmap and dendrogram based on $F_{S T}$ matrices A) with the Basque and Gascon samples and B) without them. On the right: Multidimensional scaling (MDS) based on $F_{S T}$ values A) with the Franco-Cantabrian samples and $\mathbf{B}$ ) without them. 

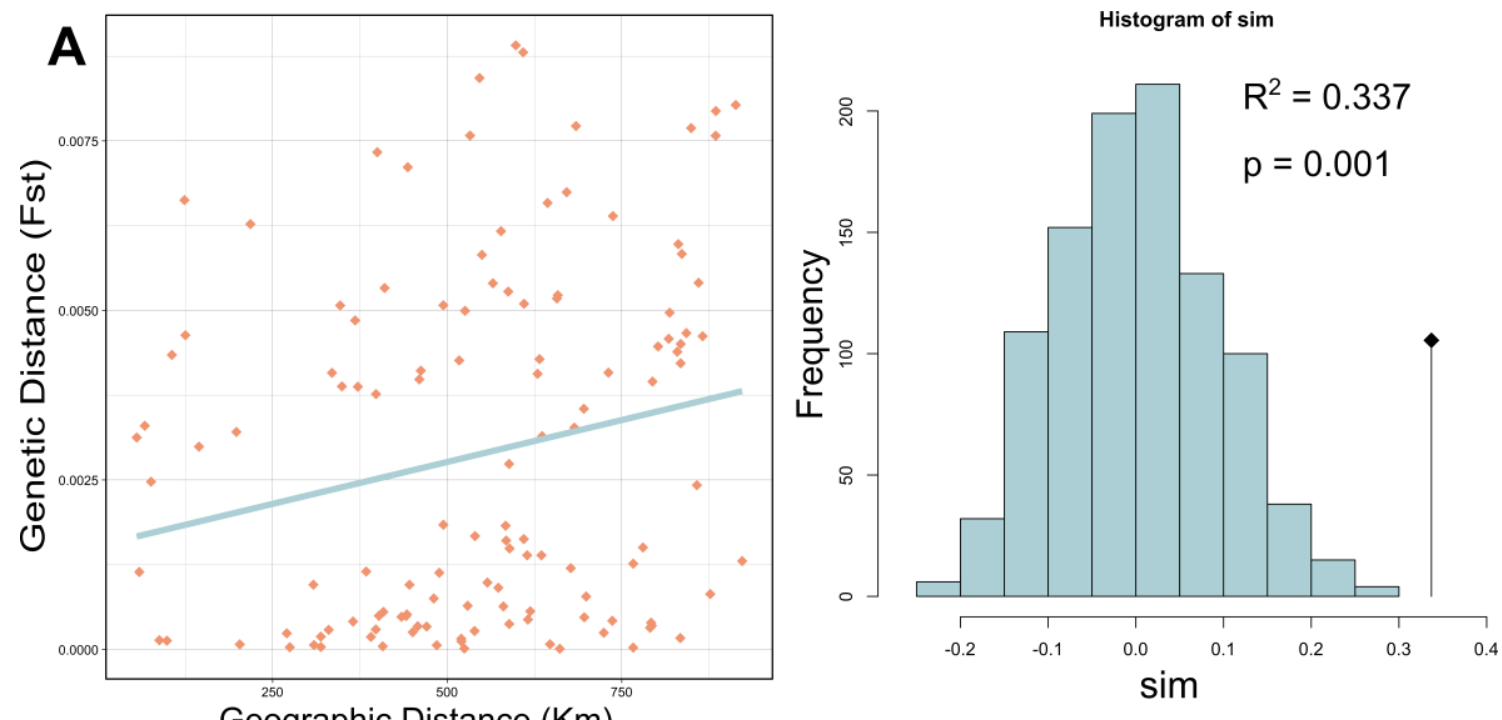

Geographic Distance (Km)
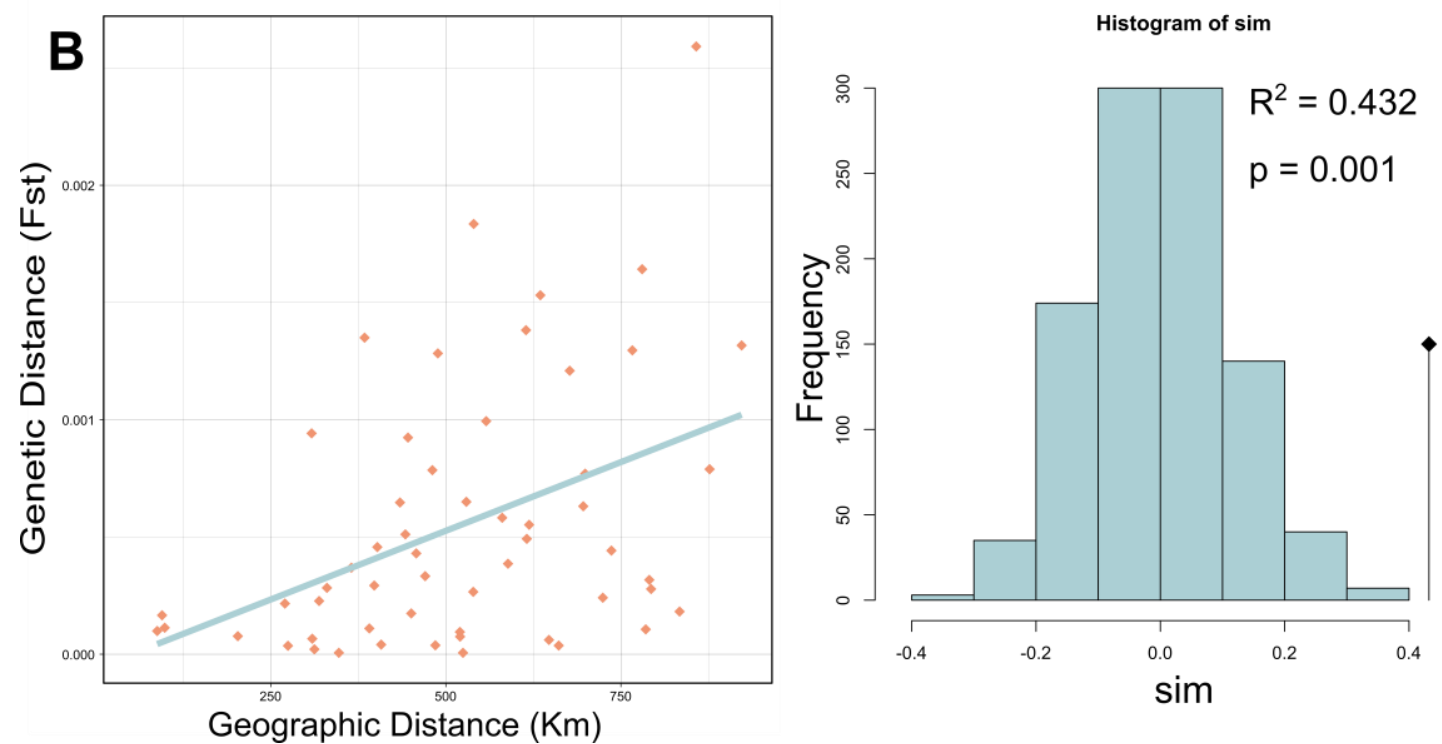

Supplementary Figure 7. Mantel test of isolation by distance between the genetic $\left(F_{S T}\right)$ and geographic (in $\mathrm{Km}$ ) distances $\mathbf{A}$ ) with the Basque and Gascon samples and $\mathbf{B}$ ) without them. $\mathbf{R}^{2}$ scores and p-values are within each figure. 


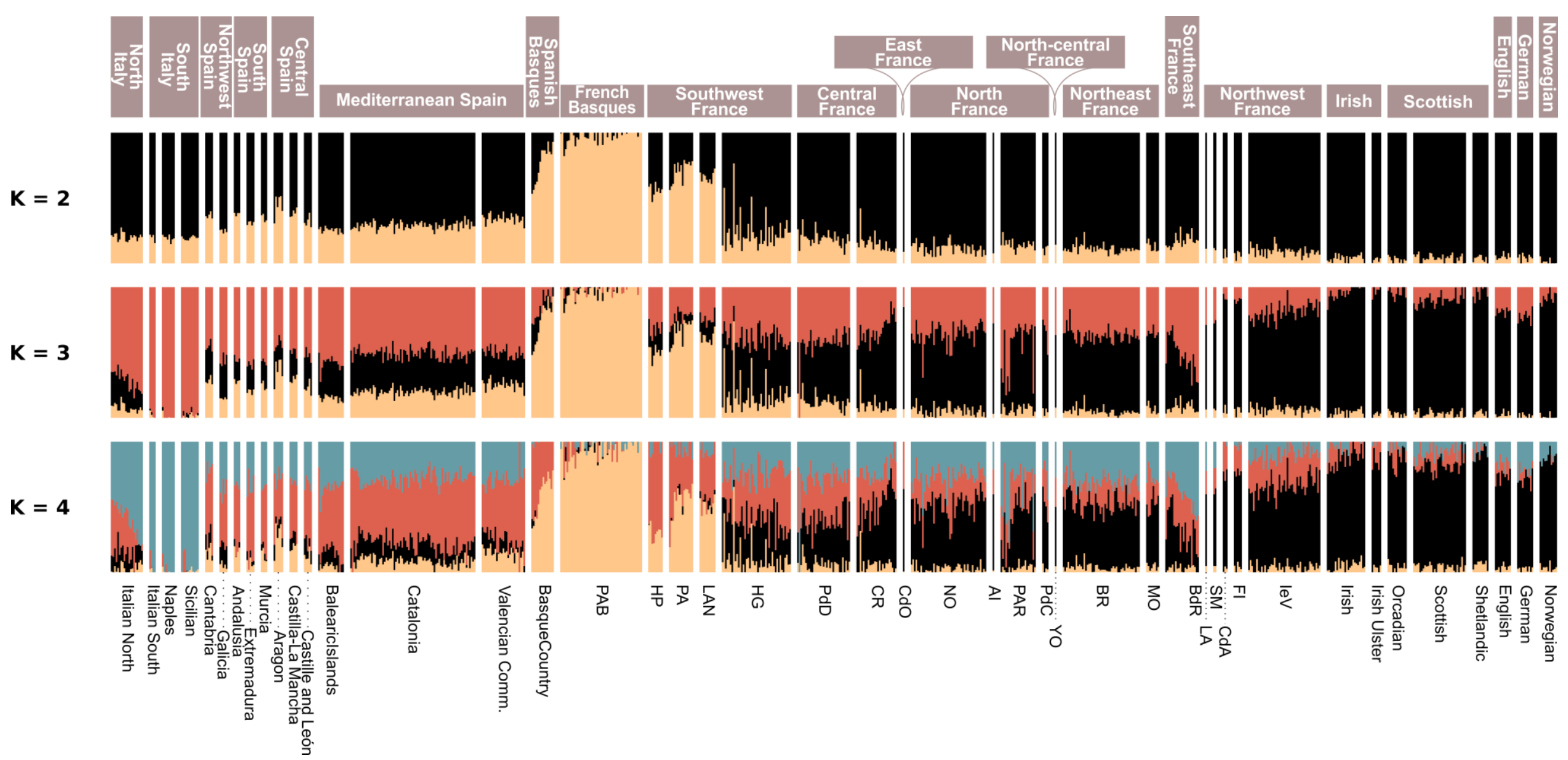

Supplementary Figure 8: ADMIXTURE results from $K=2$ to $K=4$ for the 395 French samples (Dataset A) divided in nine major groups, and 12 groups representing external sources from surrounding countries; the lowest cross-validation error was found with $\mathrm{K}=2$. 

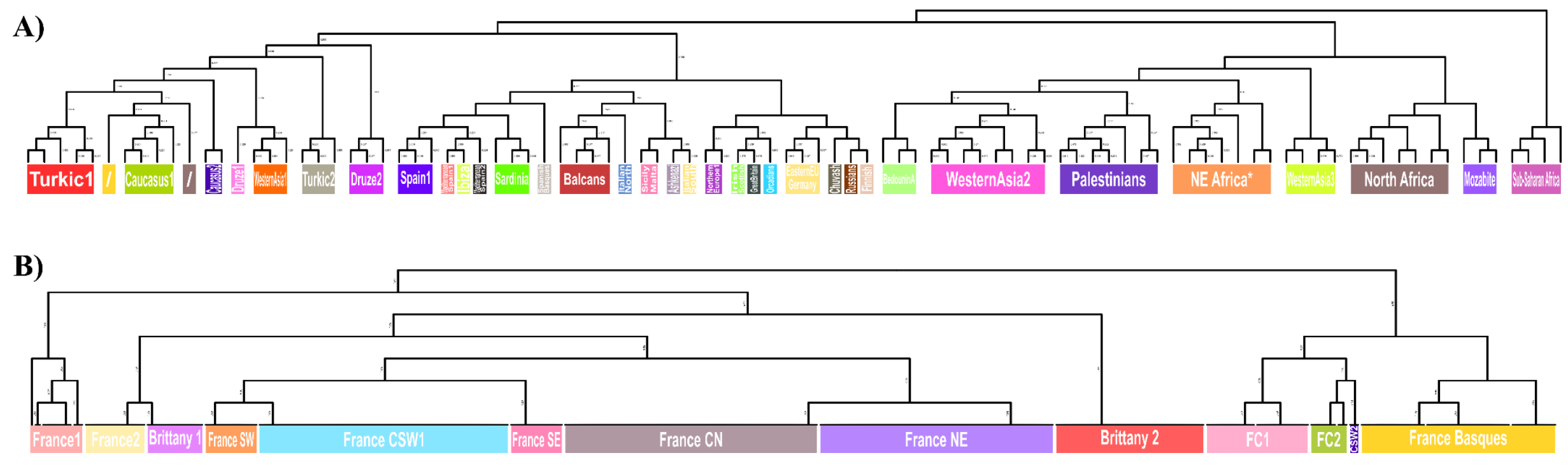

Supplementary Figure 9. A) 35 clusters detected for the external samples when France was silenced in the rerun of CromoPainter and fineSTRUCTURE. In most of the cases, clustering corresponded to the geographical origin or the linguistic affinity. Group NE Africa* was a mixture of Northern-eastern African samples plus individuals from Middle Eastern regions. Two clusters are marked with a "/" since they only included respectively one and two individuals, and were not included in the downstream analysis.B) 13 clusters detected within France using the "force file" option (-F) in fineSTRUCTURE. Groups were named based on the origin of the majority of the samples included in each cluster. Apart from France 1 and France 2, samples were distributed according to major geographical areas and, in the case of Brittany1, Brittany2, FC1, FC2, and Basques, according to more precise geographical/cultural membership. 


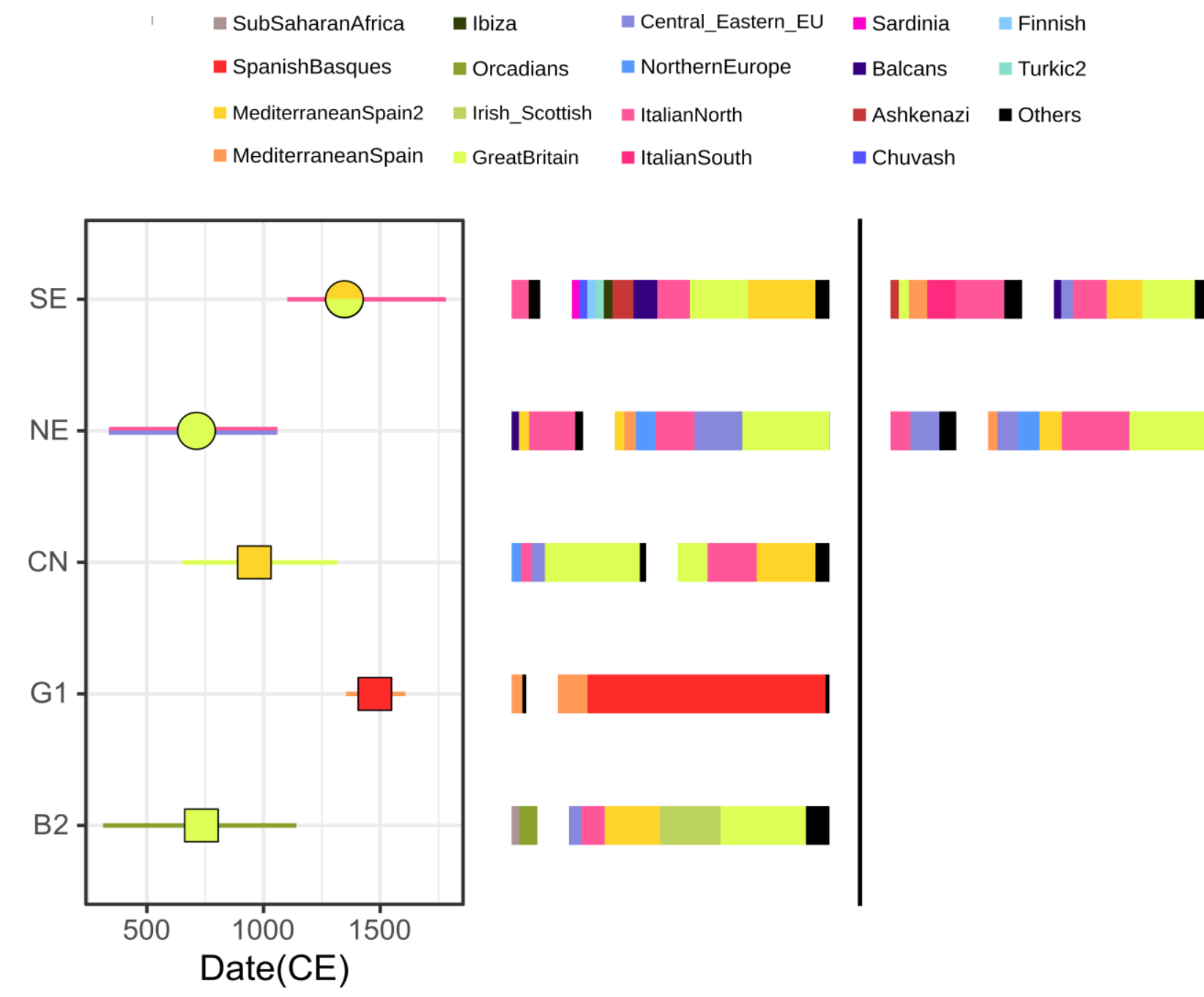

Supplementary Figure 10. Dating results for 5 French targets according to the $\mathrm{M}$ analysis in GLOBETROTTER. In the left panel, squares refer to one-date, circles to onedate-multiway. The internal color refers to the highest surrogate's value of the major source, while the color of the CI bars corresponds to the highest surrogate's value of the minor source. Sources are represented as horizontal bars on the right side and are separated by a white space (together the sources account for the $100 \%$ of the values). In the onedate-multiway cases, two different sets of sources are presented and, where needed, both colors are represented for major and minor sources. Dates have been estimated as $1950-(\mathrm{g} * \mathrm{~N})$ where $\mathrm{g}=28$ years and $\mathrm{N}$ is the calculated number of generations in the GLOBETROTTER analysis. 

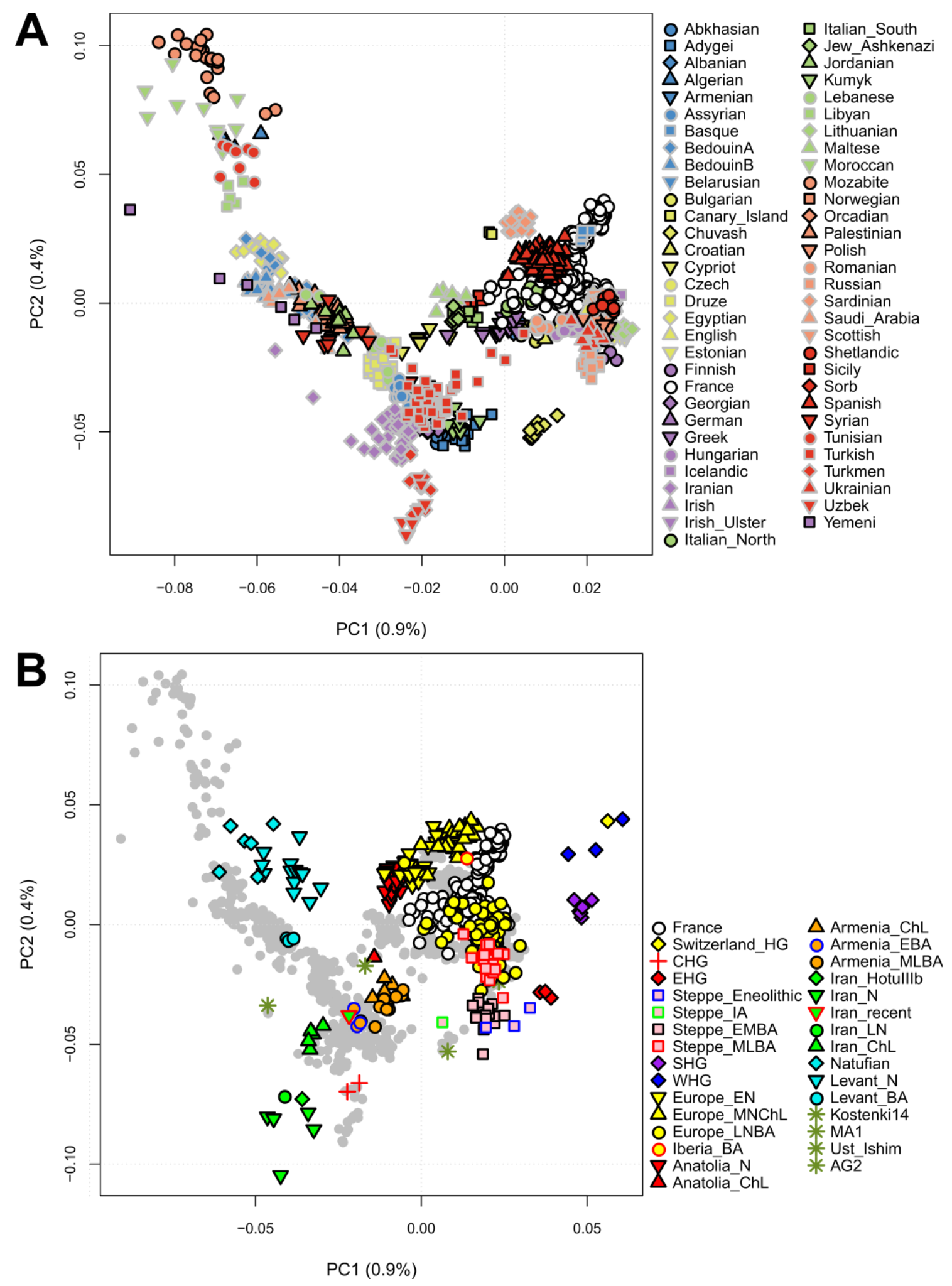

Supplementary Figure 11. Principal component analysis with dataset D. A) Only modern samples; B) Projection of ancestral populations from different periods on top of the modern samples (grey dots; among the modern populations, only France is distinguishable as white circles). 


$\begin{array}{llll}\text { Dataset Samples } & \text { Analysis } & N^{\circ} \text { of SNPs } \\ \text { A } & \mathbf{3 9 5} & \text { Allele frequency / Haplotype-based } & 142,803 / 343,884 \\ \text { B } & \mathbf{7 2 8}(395+333) & \text { Allele frequency } & 154,889 \\ \text { C } & \mathbf{1 5 2 7}(728+799) & \text { Haplotype-based } & 380,697 \\ \text { D } & 1687(1527-122+282) & \text { Allele frequency } & 163,631\end{array}$

Supplementary Table 1. Summary of the dataset composition; both number of samples and number of variants are reported according to the analysis the dataset was used for. 
Supplementary Table 2. $f 3$-statistics with the test groups being the different departments (with $\mathrm{n}>1$ ), and all possible pairs of external surrounding populations as sources; only significant Z-scores $<-3$ are reported in this table.

Supplementary Table 3. Complete list of f3-statistics with the test groups being the different departments (with $\mathrm{n}>1$ ), and all possible pairs of external surrounding populations as sources.

Supplementary note 1. Further details on the implementation of haplotype-based methods.

Extended data table. Extensive information on the French samples (department, region, area, and geographical coordinates). 